Archive for

Organic Chemistry

Arkivoc 2020, part vi, 312-329

\title{
An expedient synthesis of novel bis[thienopyridines] linked to arene or heteroarene core as novel hybrid molecules
}

\author{
Mostafa E. Salem, Ahmed H. M. Elwahy* and Ahmed F. Darweesh \\ Department of Chemistry, Faculty of Science, Cairo University, Giza 12613, Egypt \\ Email: aelwahy@hotmail.com
}

Received 07-12-2020

Accepted 08-25-2020

Published on line $09-13-2020$

\section{Abstract}

A series of novel bis(thieno[2,3-b]pyridines) based arenes or heteroarenes have been synthesized from the appropriate bis-bromoacetyl derivatives upon treatment with the corresponding 2-mercaptonicotinonitrile derivatives in ethanolic sodium ethoxide at reflux. Attempts to synthesize these compounds via bis-alkylation of the appropriate phenol derivative with the corresponding dibromo compounds using a mild base were unsuccessful.

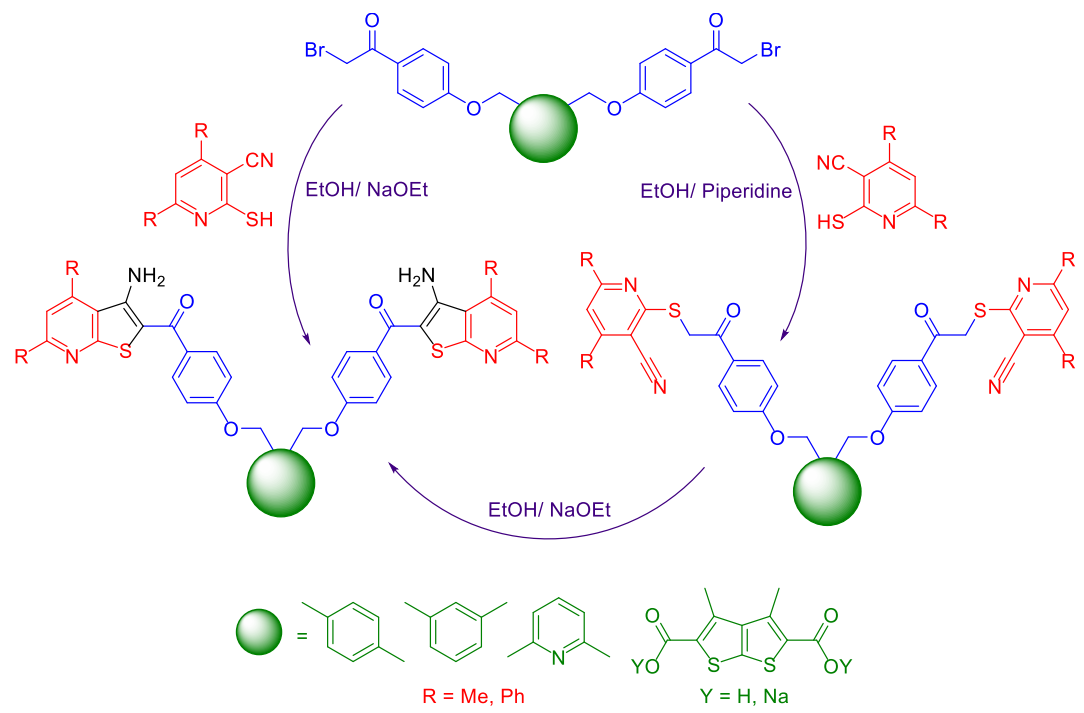

Keywords: Pyridinethiones, thieno[2,3-b]pyridines, alkylation, cyclization, bis(thieno[2,3- $b]$ pyridines). 


\section{Introduction}

Pyridine is one of the most interesting heterocyclic rings due to their wide range of pharmaceutical properties including anti-inflammatory, ${ }^{1,2}$ antiasthmatic, ${ }^{3}$ antidepressant, ${ }^{4}$ acetylcholinesterase inhibitors (AChE), ${ }^{5}$ HIV protease inhibitors. ${ }^{6}$ They are also used for treating hypertension ${ }^{7}$ or hypotension, ${ }^{8}$ as well as for inducing or preventing apoptosis. ${ }^{9}$

The pyridine structure is present in many natural compounds like nicotinic acid (vitamin B3) and pyridoxine (vitamin B6) and also in many drugs (Figure 1).

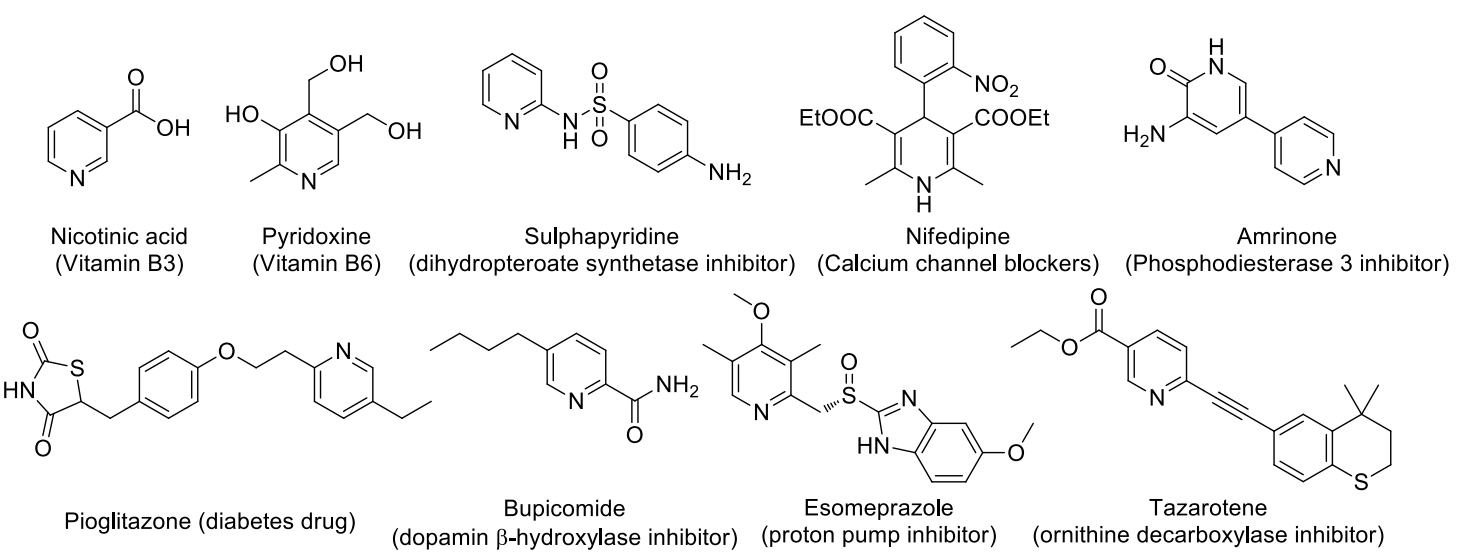

Figure 1. Natural compounds and drugs containing pyridine ring.

Pyridines are also exploited in agrochemistry ${ }^{10,11}$ as well as in materials science. ${ }^{12}$ Different methodologies for construction and functionalization of pyridine derivatives have been recently reviewed. ${ }^{13,14}$ Thienopyridines are considered as one of the important fused heterocyclic compounds for their usefulness therapeutic applications. ${ }^{15,16}$

Moreover, several thienopyridine derivatives are known to possess antiviral, ${ }^{17}$ anti-inflammatory, ${ }^{18}$ antimicrobial, ${ }^{19}$ antidiabetic, ${ }^{20}$ antihypertensive ${ }^{21}$ and osteogenic activities. ${ }^{22}$ In particular, thieno[2,3-b]pyridines are reported to be useful as anticancer agents. ${ }^{23-25}$

Due to the numerus applications of pyridine as well as thienopyridine systems, exploring novel structures and novel synthetic procedures for this class of compounds is still in need.

Motivated by these findings and in conjunction with recent interest on molecular hybridization concept as well as on the chemistry of bis-heterocyclic compounds, ${ }^{26-39}$ we report herein on the synthesis of novel bis[thienopyridines] linked to arene or heteroarene spacers.

\section{Results and Discussion}

The new bis(thieno[2,3-b]pyridines) 5(a-d), in which the two thienopyridine moieties are linked via aromatic spacers, have been synthesized from the appropriate bis-bromoacetyl derivatives $\mathbf{2}(\mathbf{a}-\mathbf{b})$ upon treatment with the corresponding 2-mercaptonicotinonitrile derivatives ${ }^{34} \mathbf{3} \mathbf{a}$ and $\mathbf{3} \mathbf{b}$ in ethanolic sodium ethoxide at reflux. Compounds $\mathbf{2}(\mathbf{a}-\mathbf{b})$ were obtained by bromination of the appropriate bis(acetyl) derivatives $\mathbf{1}(\mathbf{a}-\mathbf{b})^{40}$ upon treatment with $\mathrm{N}$-bromosuccinimide in acetonitrile at reflux. ${ }^{40}$ 
The step-wise synthesis of $\mathbf{5}(\mathbf{a}-\mathbf{d})$ was also performed via initial formation of bis(sulfanediyl)) bis(nicotinonitriles) $\mathbf{4}(\mathbf{a}-\mathbf{d})$ by the reaction of $\mathbf{2}(\mathbf{a}-\mathbf{b})$ with $\mathbf{2}$-mercaptonicotinonitriles $\mathbf{3 a}$ and $\mathbf{3 b}$ in ethanol containing few drops of piperidine at reflux. Cyclization of the latter compounds to the corresponding bis(thienopyridines) $\mathbf{5}(\mathbf{a}-\mathbf{d}$ ) was achieved in $65-77 \%$ yields, upon heating at reflux in ethanolic solution containing sodium ethoxide (Scheme 1).

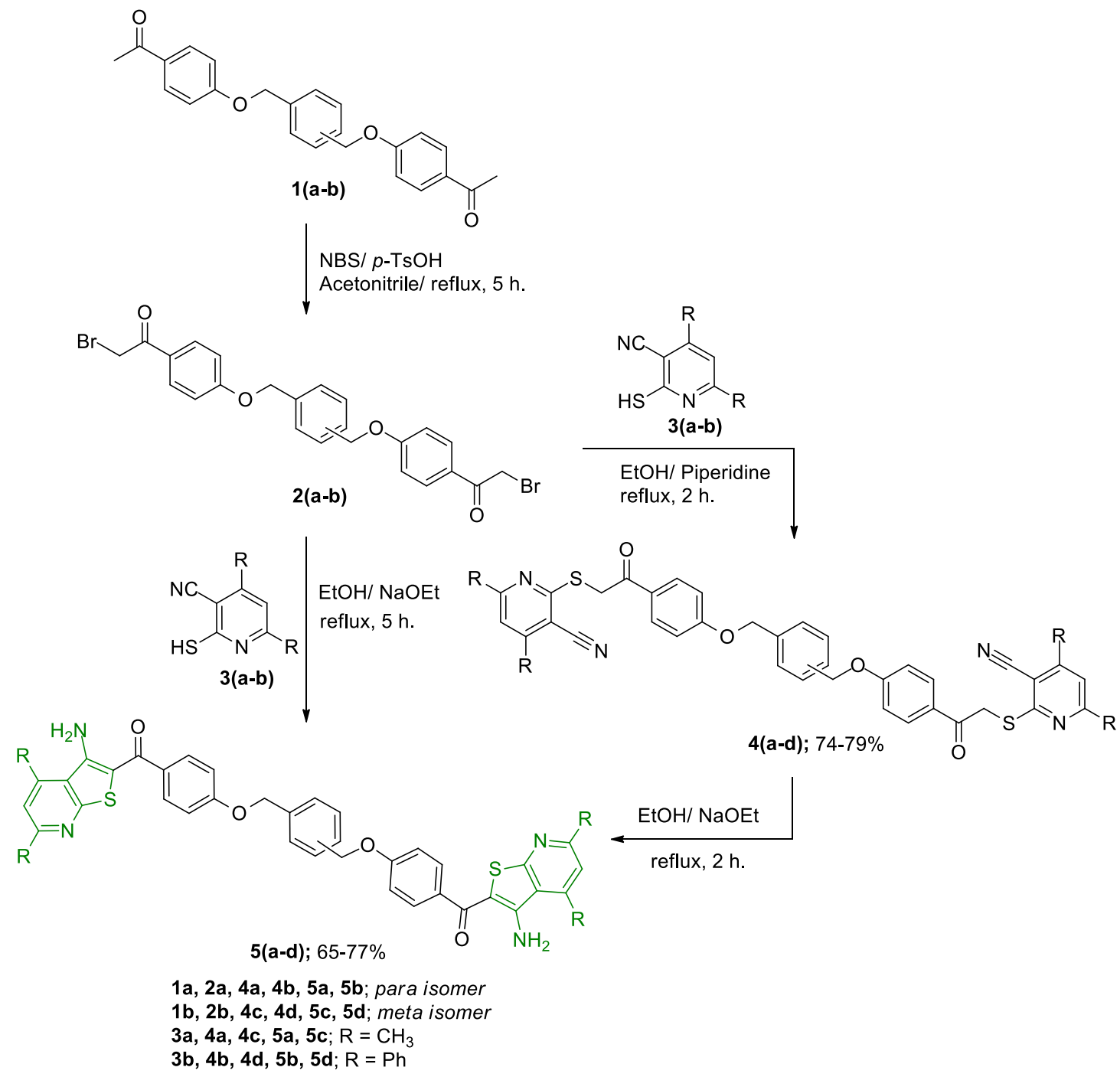

Scheme 1. Synthesis of bis(thieno[2,3-b]pyridines) $\mathbf{5 ( a - d ) ~ l i n k e d ~ v i a ~ a r o m a t i c ~ s p a c e r s . ~}$

The structures of compounds 5(a-d) were established based on their spectral data and elemental analyses. Thus, compound 5c showed in its IR spectra the presence of absorption bands at 3480, 3436 and $1593 \mathrm{~cm}^{-1}$ characteristic for the amino group and the carbonyl band, respectively. The successful ring closure of $\mathbf{4 c}$ is confirmed by the disappearance of absorption bands characteristic for the cyano group in compound $\mathbf{5 c}$ together with the presence of this band in compound 4c. Moreover, the ${ }^{1} \mathrm{H}$ NMR spectra provide a further confirmation for the ring closure of $\mathbf{4 c}$ to $\mathbf{5 c}$ Thus, compound $\mathbf{4 c}$ exhibited the presence of $\mathrm{SCH}_{2} \mathrm{protons}$ resonated at $\delta 4.77 \mathrm{ppm}$ as a singlet signal integrating four protons in compound $4 \mathrm{c}$ while that of compound 5c did not show this signal. Moreover, compounds $\mathbf{4 c}$ and $\mathbf{5 c}$ also featured the methylene ether linkage $\mathrm{OCH}_{2}$ as a singlet signal at $\delta 5.2 \mathrm{ppm}$. All other protons were seen at the expected chemical shifts and integral values (See experimental section). 
The same methodology can also be applied for the synthesis of (((pyridine-2,6diylbis(methylene))bis(oxy))bis(4,1-phenylene))bis((3-aminothieno[2,3-b]pyridin-2-yl)meth-anones) 11a and 11b starting from 1,1'-(((pyridine-2,6-diylbis(methylene))bis(oxy))bis(4,1-phenylene))diethanone (8). Thus, reaction of of 2,6-bis(bromomethyl)pyridine (7) with potassium 4-acetylphenolate in DMF at reflux afforded 8 in $78 \%$ yield. Subsequent bromination of $\mathbf{8}$ upon treatment with $N$-bromosuccinimide in acetonitrile at reflux afforded bis(bromoacetyl) product 9 in 72\% yield. It is worthy to mention that bis(bromoacetyl) products 9 as well as $\mathbf{2} \mathbf{a}$ and $\mathbf{2} \mathbf{b}$ were isolated as pure single products while no aromatic brominated products under the reaction conditions were detected. Reaction of $\mathbf{9}$ with 2-mercaptonicotinonitriles $\mathbf{3 a}$ and $\mathbf{3 b}$ in ethanolic solution containing piperidine at reflux gave the corresponding bis(sulfanediyl)bis(nicotinenitriles) 10a and 10b which could then be cyclized to the corresponding bis(thienopyridines) 11a and 11b upon heating at reflux in ethanolic sodium ethoxide solution (Scheme 2). Reaction of $\mathbf{9}$ with each of $\mathbf{3 a}$ and $\mathbf{3 b}$ in ethanolic sodium ethoxide at reflux gave the desired bipodals $11 \mathbf{a}$ and $\mathbf{1 1 b}$ in $70 \%$ and $71 \%$ yields, respectively.

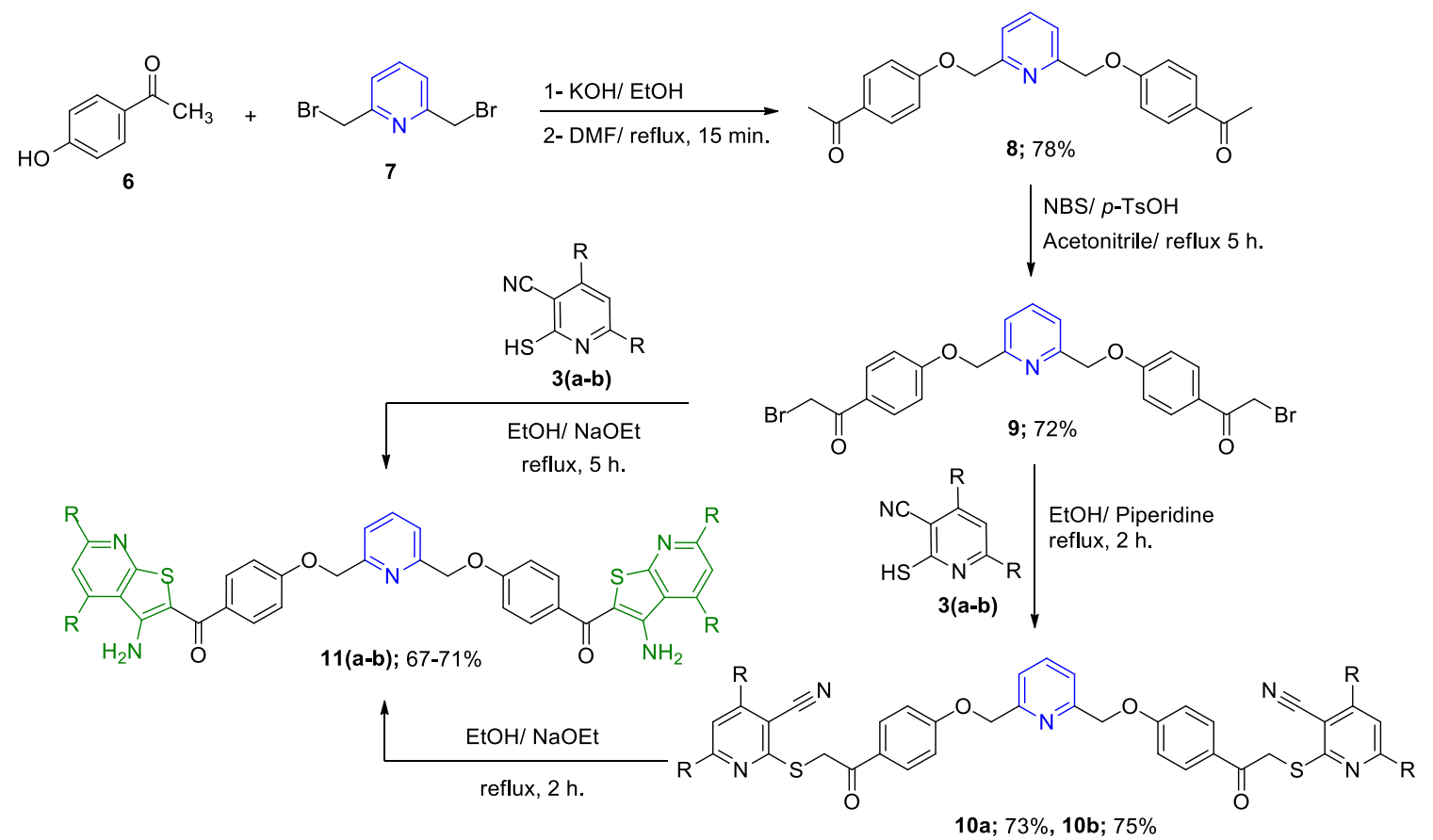

3a, 10a, 11a; $\mathrm{R}=\mathrm{CH}_{3}$

$3 b, 10 b, 11 b ; R=P h$

Scheme 2. Synthesis of bis(thieno[2,3-b]pyridines) $\mathbf{1 1 ( a - b ) ~ l i n k e d ~ v i a ~ p y r i d i n e ~ s p a c e r s . ~}$

The study was extended to include the synthesis of novel bis(thieno[2,3-b]pyridines) 15(a-b) which are linked to thienothiophene core (Scheme 3). The bis-bromoacetyl derivative $13^{41}$ was chosen as precursor and was obtained as previously reported from the corresponding bis(acetyl) derivative $12^{41}$ upon treatment with $\mathrm{N}$-bromosuccinimide in acetonitrile at reflux. ${ }^{41}$

Both of two-step and one-step syntheses of $\mathbf{1 5}(\mathbf{a}-\mathbf{b})$ were performed. Thus, reaction of 13 with 2mercaptonicotinonitrile derivatives $\mathbf{3 a}$ and $\mathbf{3 b}$ in ethanolic sodium ethoxide at reflux afforded the corresponding sodium 3,4-bis((4-(3-aminothieno[2,3-b]pyridine-2-carbonyl)phenoxy)-methyl)thieno[2,3b] thiophene-2,5-dicarboxylate 15(a-b) in 69-72\% yields. On the other hand, step-wise synthesis of 15(a-b) was achieved by firstly formation of diethyl 3,4-bis((4-(2-(3-cyanopyridin-2-ylthio)acetyl)phenoxy)methyl)thieno[2,3-b]thiophene-2,5-dicarboxylate $\mathbf{1 4}(\mathbf{a}-\mathrm{b})$ by the reaction of 13 with 2- 
mercaptonicotinonitriles $\mathbf{3}(\mathbf{a}-\mathbf{b})$ in ethanol containing few drops of piperidine at reflux. Subsequent heating of 14(a-b) at reflux in ethanolic solution containing sodium ethoxide afforded the corresponding sodium 3,4bis((4-(3-aminothieno[2,3-b]pyridine-2-carbonyl)phenoxy)methyl)thieno[2,3-b]thiophene-2,5-dicarboxylate 15(a-b) in 67 and 69\% yields, respectively. The free dicarboxylic acid, 3,4-bis((4-(3-amino-4,6diphenylthieno[2,3-b]pyridine-2-carbonyl)phenoxy)methyl)thieno[2,3-b]thiophene-2,5-dicarboxylic acid (16) was liberated upon neutralization of $\mathbf{1 5 b}$ with hydrochloric acid (Scheme 3).

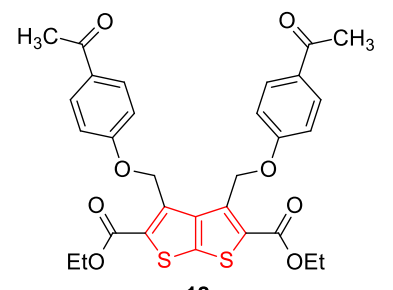

12

$\mathrm{NBS} / p-\mathrm{TsOH}$

$\mathrm{CH}_{3} \mathrm{CN}$, reflux $5 \mathrm{~h}$.

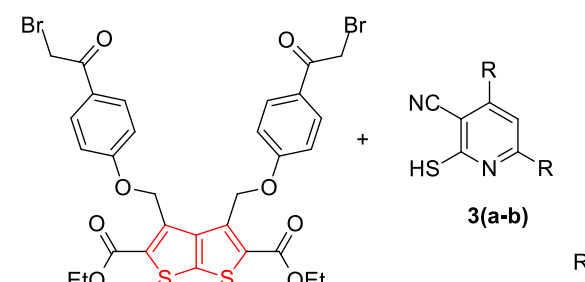

13
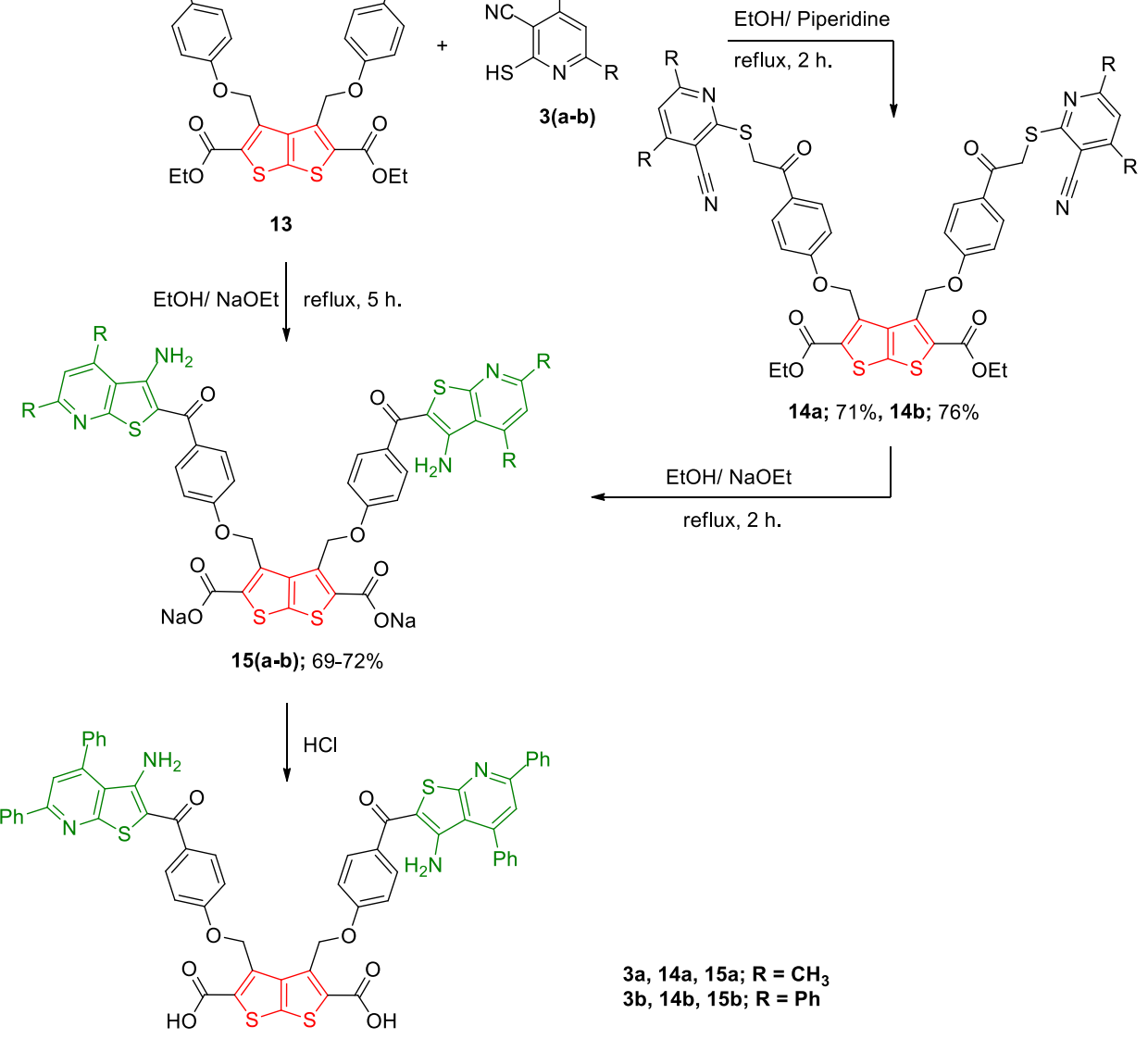

$3 \mathrm{a}, 14 \mathrm{a}, 15 \mathrm{a} ; \mathrm{R}=\mathrm{CH}_{3}$

$3 b, 14 b, 15 b ; R=P h$

$16 ; 66 \%$

Scheme 3. Synthesis of bis(thieno[2,3-b]pyridines) 15(a-b) linked via thienothiophene spacers.

Compound 14a featured the ethyl ester protons as triplet and quartet signals at $\delta 1.27$ and 4.33 in their ${ }^{1} \mathrm{H}$ NMR spectra. On the other hand, these signals disappeared in the ${ }^{1} \mathrm{H} N M R$ spectra of both of the di-sodium salts $\mathbf{1 5}(\mathbf{a}-\mathbf{b})$ as well as the free dicarboxylic acid $\mathbf{1 6}$. The latter compound showed the presence of broad signal 
at $\delta 3.40$ characteristic for the $\mathrm{COOH}$ proton. The structure of $\mathbf{1 6}$ was further confirmed by IR spectra which showed absorption ban at $3430 \mathrm{~cm}^{-1}$ characteristic for the $\mathrm{OH}$ of the carboxylic acid $\mathrm{COOH}$.

Our study included also the synthesis of novel sodium 3,4-bis((2-(3-aminothieno[2,3-b]pyridine-2carbonyl)phenoxy)methyl)thieno[2,3-b]thiophene-2,5-dicarboxylate 20(a-b) as outlined in Scheme 4. For this purpose, the bis-bromoacetyl derivative $18^{41}$ was obtained from the corresponding bis(acetyl) derivative $17^{41}$ upon treatment with $\mathrm{N}$-bromosuccinimide in acetonitrile at reflux. ${ }^{41}$ Reaction of 18 with 2mercaptonicotinonitrile derivatives $\mathbf{3} \mathbf{a}$ and $\mathbf{3} \mathbf{b}$ in ethanol containing few drops of piperidine at reflux afforded the corresponding diethyl 3,4-bis((2-(2-((3-cyano-4,6-dimethylpyridin-2-yl)thio)acetyl)phenoxy)methyl)thieno[2,3-b]thiophene-2,5 dicarboxylate $\mathbf{1 9}(\mathbf{a}-\mathbf{b})$ in 71 and $77 \%$ yields, respectively. Subsequent heating of 19(a-b) at reflux in ethanolic solution containing sodium ethoxide did not lead to the formation of sodium 3,4bis((2-(3-aminothieno[2,3-b]pyridine-2-carbonyl)phenoxy)methyl)thieno[2,3-b]thiophene-2,5-dicarboxylate 20(a-b) but gave instead the corresponding sodium 3,4-bis(3-(3-amino-thieno[2,3-b]pyridin-2-yl)benzofuran-2$\mathrm{yl}$ )thieno[2,3-b]thiophene-2,5-dicarboxylates $\mathbf{2 1 ( a - b ) . ~ T h e ~ l a t t e r ~ c o m p o u n d s ~ c o u l d ~ a l s o ~ o b t a i n e d ~ i n ~ o n e ~ s t e p ~}$ by the reaction of bis-bromoacetyl derivative $\mathbf{1 8}$ with 2-mercaptonicotinonitrile derivatives $\mathbf{3 a}$ and $\mathbf{3 b}$ in ethanolic sodium ethoxide at reflux (Scheme 4).
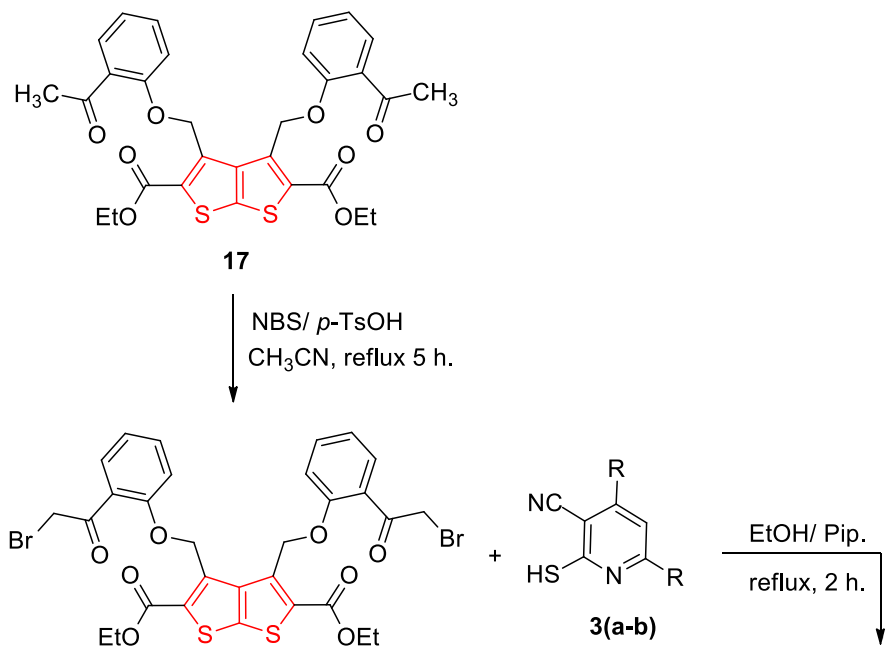

18
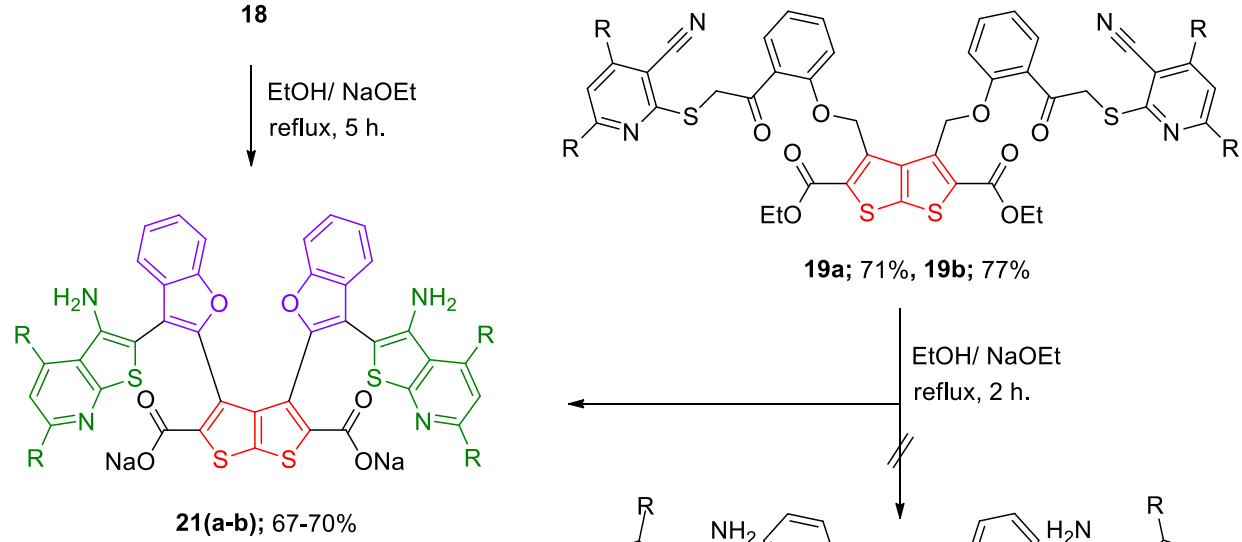

3a, 19a, 20a, 21a; $\mathbf{R}=\mathrm{CH}_{3}$ 3b, 19b, 20b, 21b; R = Ph

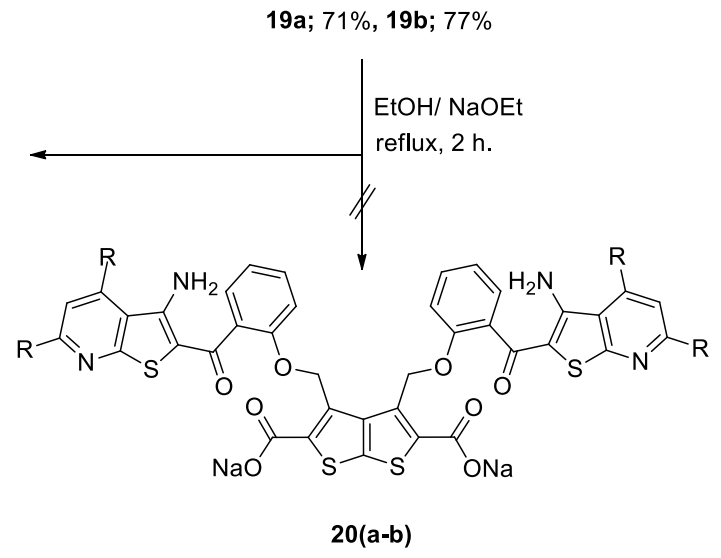

Scheme 4. Synthesis of bis(thieno[2,3-b]pyridines) 20(a-b). 
The formation of $\mathbf{2 1 a}$ and $\mathbf{2 1} \mathbf{b}$ proceeded via intramolecular cyclocondensation of the methylene groups at the 3-position of thienothiophene with the ketonic groups of compound 19a and 19b, respectively. Similar behavior of some related systems has been previously reported by us as well as by other groups. ${ }^{42-45}$

The structures of compounds $\mathbf{2 1 ( a - b ) ~ w e r e ~ e s t a b l i s h e d ~ b a s e d ~ o n ~ t h e i r ~ s p e c t r a l ~ d a t a ~ a n d ~ e l e m e n t a l ~}$ analyses. Thus compound 21a showed in its IR spectrum the presence of absorption bands at 3432 and 3330 $\mathrm{cm}^{-1}$ characteristic for the amino group. Moreover, its ${ }^{1} \mathrm{H}$ NMR spectrum provide a further confirmation for the ring closure of 19a to 21a Thus, compound 21a did not revealed the presence of characteristic signals for the methylene ether protons $\mathrm{SCH}_{2}$ as well as the $\mathrm{OCH}_{2}$ protons. On the other hand, its precursor 19a showed two singlet signals each integrating four protons resonated at $\delta 4.33$ and $5.67 \mathrm{ppm}$ characteristic for the $\mathrm{SCH}_{2}$ and the $\mathrm{OCH}_{2}$ protons, respectively. All other protons were seen at the expected chemical shifts and integral values (See experimental section).

Motivated by these results, we also attempted to synthesize the novel bis(thieno[2,3-b]pyridines) 22 and 23 (Figure 2) which are linked to pyridine and quinoxaline, respectively, as spacers by the reaction of 2mercaptonicotinonitrile $\mathbf{3}(\mathbf{a}-\mathbf{b})$ with the appropriate bis(bromoacetyl) derivatives using a similar approach.

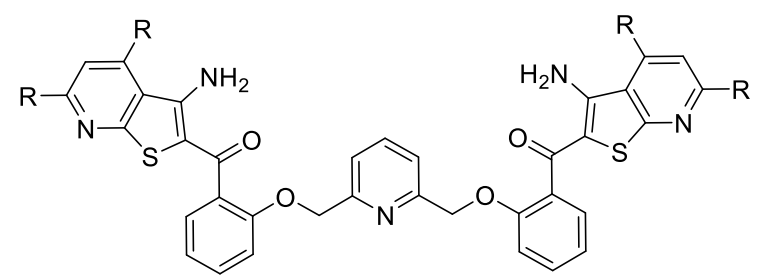

22

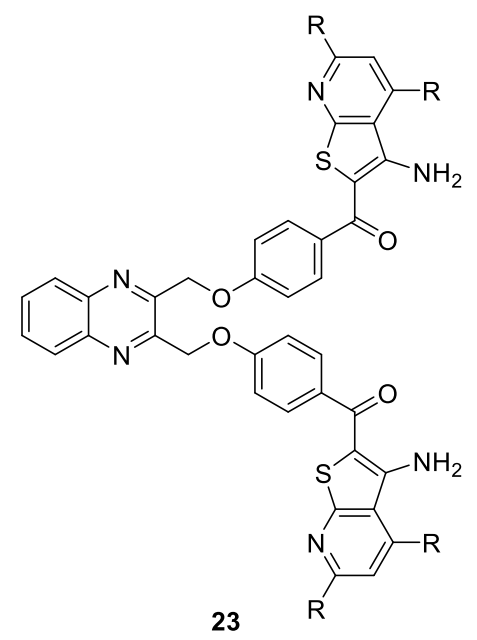

23

Figure 2. Structures of bis(thieno[2,3-b]pyridines) linked to pyridine and quinoxaline 22 and 23.

To achieve this goal we studied the synthesis of bis(acetyl)pyridine $\mathbf{2 5}$ by the reaction of 2,6bis(bromomethyl)pyridine (7) with potassium 2-acetylphenolate in DMF at reflux. Likewise, bis(acetyl)quinoxaline $\mathbf{2 8}$ were also obtained by the reaction of 2,3-bis(bromomethyl)quinoxalines (27) with potassium 4-acetylphenolate in DMF at reflux. Attempted bromination of $\mathbf{2 5}$ and $\mathbf{2 8}$ to give the corresponding bis(bromoacetyl) derivatives 26 and 29, respectively, either by reaction with $\mathrm{Br}_{2}$ in acetic acid or upon treatment with $\mathrm{N}$-bromosuccinimide in acetonitrile at reflux were unsuccessful. In all trials, the reactions gave a mixture of products that were not easily handled and have not been characterized as yet (Scheme 5).

Aiming at synthesizing compounds 22 and 23, which could not prepared by the above method, we then turned to another strategy. For this purpose, (3-amino-4,6-dimethylthieno[2,3-b]pyridin-2-yl)(4hydroxyphenyl)methanone $(\mathbf{3 1})^{46}$ have initially been synthesized by the reaction of 2-bromo-1-(4hydroxyphenyl)ethanone (30) with $\mathbf{3 a}$. Compound $\mathbf{3 1}$ was then allowed to react with each of bis(bromomethylquinoxaline) $\mathbf{7}$ and $\mathbf{2 7}$ in the presence of ethanolic solution containing sodium ethoxide aiming at obtaining $\mathbf{2 2}$ and $\mathbf{2 3}$, respectively. Unfortunately, the ${ }^{1} \mathrm{H}-\mathrm{NMR}$ of the reaction products indicated the presence of the target compounds $\mathbf{2 2}$ and $\mathbf{2 3}$ together with other non-isolable products which may be formed 
as a result of competing $N$-alkylation reaction (Scheme 6). It is worthy mentioned that repeated attempts to get the target products $\mathbf{2 2}$ or $\mathbf{2 3}$ by carrying out the alkylation reactions under different basic conditions were also unsuccessful.
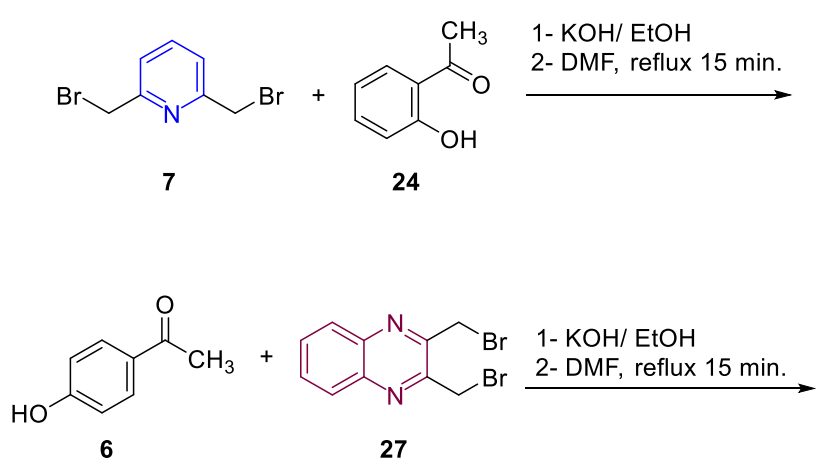

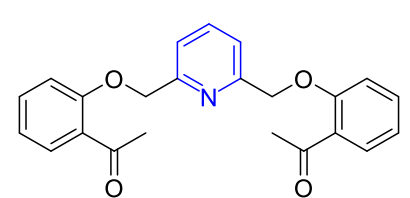

$25 ; 76 \%$

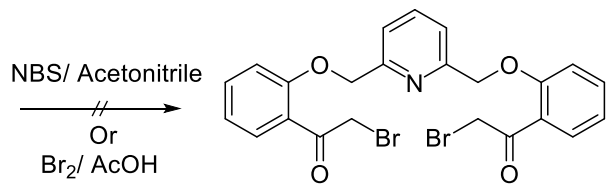

26

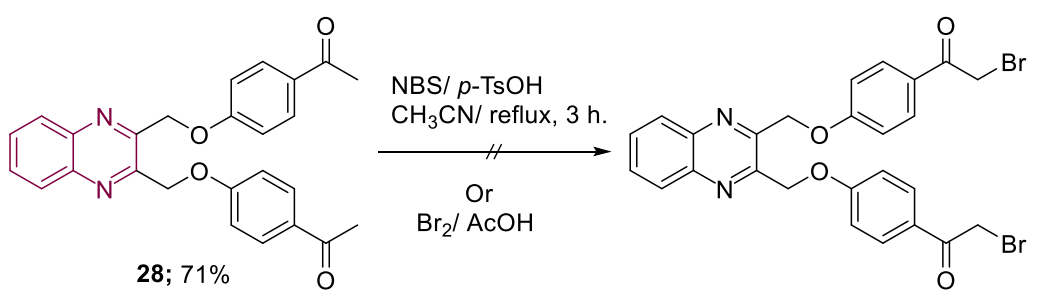

29

Scheme 5. Attempted Synthesis of bis(bromoacetyl) derivatives 26 and 29.<smiles>Cc1cc(C)c(C#N)c(S)n1</smiles>

3a<smiles>BrCCBr</smiles>

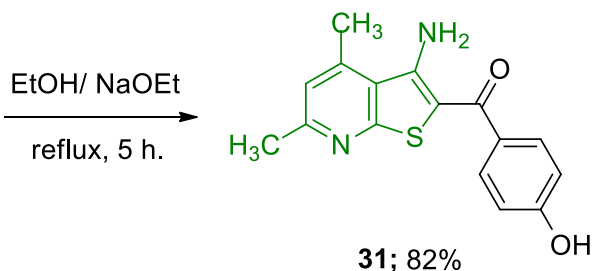

1- $\mathrm{KOH} / \mathrm{EtOH}$

2- DMF, reflux $15 \mathrm{~min}$.

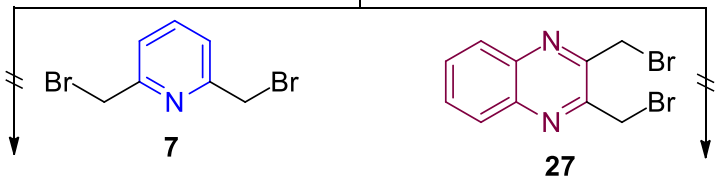

22

Scheme 6. Attempts to Synthesis of bis(thieno[2,3-b]pyridines) 22 and 23.

\section{Conclusions}

We developed an efficient synthesis of previously unreported bis(thienopyridines) which are linked to arene or heteroarene via phenoxymethyl groups. The structures of the newly synthesized compounds were full characterized by both spectral data and elemental analyses. Trials to synthesize these compounds by alkylation of thieno[2,3-b]pyridin-2-yl)(4-hydroxyphenyl)methanone with the appropriate dibromocompounds were unsuccessful. The main advantages of these reactions include mild reaction conditions, good yields, easily accessible starting materials and straightforward product isolation. The newly synthesized 
compounds achieved the "hybrid molecules" concept which aims at combining two promising pharmacophores in one molecule.

\section{Experimental Section}

General. Melting points were determined in open glass capillaries with a Gallenkamp apparatus and were not corrected. Infrared spectra were recorded in potassium bromide disks on a PyeUnicam SP3-300 and Shimadzu FTIR 8101 PC infrared spectrophotometer. NMR spectra were recorded with a Varian Mercury VXR-300 NMR spectrometer operating at $300 \mathrm{MHz}\left({ }^{1} \mathrm{H} N M R\right)$ and $75 \mathrm{MHz}\left({ }^{13} \mathrm{C} \mathrm{NMR}\right)$, a Varian VXR spectrometer operating at $400 \mathrm{MHz}\left({ }^{1} \mathrm{H}\right.$ NMR) and $101 \mathrm{MHz}\left({ }^{13} \mathrm{C} \mathrm{NMR}\right)$ and a Varian VXR spectrometer operating at $500 \mathrm{MHz}\left({ }^{1} \mathrm{H} \mathrm{NMR}\right)$ and $126 \mathrm{MHz}\left({ }^{13} \mathrm{C} N M R\right)$ using TMS as an internal standard and DMSO- $d_{6}$ as a solvent. Mass spectra were measured on a GC MS-QP1000 EX spectrometer at $70 \mathrm{eV}$. Elemental analyses were carried out at the Microanalytical Center of Cairo University, Giza, Egypt. The starting $\mathbf{1}(\mathbf{a}-\mathbf{b}),{ }^{40} \mathbf{2}(\mathbf{a}-\mathbf{b}),{ }^{40} \mathbf{3}(\mathbf{a}-\mathbf{b}),^{34} \mathbf{1 2},{ }^{41} \mathbf{1 3},{ }^{41} \mathbf{1 7},,^{41}$ $1 \mathbf{8}^{41}$ and $31^{46}$ were prepared following literature procedures.

Synthesis of 2,2'-(2,2'-(4,4'-(phenylenebis(methylene))bis(oxy)bis(4,1-phenylene))bis(2-oxoethane-2,1-

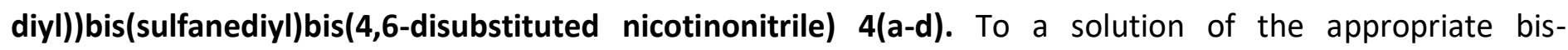
bromoacetyls $\mathbf{2 a}$ or $\mathbf{2 b}(5 \mathrm{mmol})$ in ethanol $(25 \mathrm{~mL})$ containing $2-3$ drops piperidine, the appropriate 2mercapto-4,6-disubstituted nicotinonitriles $\mathbf{3 a}$ or $\mathbf{3 b}(10 \mathrm{mmol})$ was added. The reaction mixture was heated at reflux for 3-4 $\mathrm{h}$. The solid product obtained upon cooling was filtered off and recrystallized from ethanol/ DMF to afford the title compounds $4(a-d)$.

2,2'-(2,2'-(4,4'-(1,4-Phenylenebis(methylene))bis(oxy)bis(4,1-phenylene))bis(2-oxoethane-2,1-

diyl))bis(sulfanediyl)bis(4,6-dimethylnicotinonitrile) (4a). Pale yellow powder, $\mathrm{mp} 227-229{ }^{\circ} \mathrm{C}$; yield $78 \%$, IR $\left(\mathrm{KBr}\right.$ disc) $v=3064,2948,2215,1678,1599,1422 \mathrm{~cm}^{-1} .{ }^{1} \mathrm{H}$ NMR $(300 \mathrm{MHz}, \mathrm{DMSO}) \delta 2.19\left(\mathrm{~s}, 6 \mathrm{H}, \mathrm{CH}_{3}\right), 2.39(\mathrm{~s}$, $\left.6 \mathrm{H}, \mathrm{CH}_{3}\right), 4.77\left(\mathrm{~s}, 4 \mathrm{H}, \mathrm{SCH}_{2}\right), 5.25\left(\mathrm{~s}, 4 \mathrm{H}, \mathrm{OCH}_{2}\right), 7.04(\mathrm{~s}, 2 \mathrm{H}$, pyridine-5-H), $7.15(\mathrm{~d}, 4 \mathrm{H}, J 8.7 \mathrm{~Hz}), 7.50(\mathrm{~s}, 4 \mathrm{H}$, $\mathrm{ArH}), 8.04(\mathrm{~d}, 4 \mathrm{H}, \mathrm{J} 8.7 \mathrm{~Hz})$. MS (m/z) $698\left(\mathrm{M}^{+}\right)$. Anal. Calcd. For $\mathrm{C}_{40} \mathrm{H}_{34} \mathrm{~N}_{4} \mathrm{O}_{4} \mathrm{~S}_{2}$ : C, 68.75; $\mathrm{H}, 4.90 ; \mathrm{N}, 8.02 ; \mathrm{S}, 9.18$. Found: $\mathrm{C}, 68.48 ; \mathrm{H}, 4.79 ; \mathrm{N}, 8.19 ; \mathrm{S}, 9.24$.

2,2'-(2,2'-(4,4'-(1,4-Phenylenebis(methylene))bis(oxy)bis(4,1-phenylene))-bis(2-oxoethane-2,1-

diyl))bis(sulfanediyl)bis(4,6-diphenylnicotinonitrile) (4b). Pale yellow powder, $\mathrm{mp} 231-233{ }^{\circ} \mathrm{C}$; yield $79 \%$, IR $\left(\mathrm{KBr}\right.$ disc) $v=3057,2917,2214,1686,1597,1461 \mathrm{~cm}^{-1} .{ }^{1} \mathrm{H} \mathrm{NMR}(300 \mathrm{MHz}, \mathrm{DMSO}) \delta 4.79\left(\mathrm{~s}, 4 \mathrm{H}, \mathrm{SCH}_{2}\right), 5.34(\mathrm{~s}$, $4 \mathrm{H}, \mathrm{OCH}_{2}$ ), $7.06(\mathrm{~s}, 2 \mathrm{H}$, pyridine-5-H), 7.09-7.43 (m, 10H, ArH), 7.53-7.82 (m, 10H, ArH), $7.85(\mathrm{~s}, 4 \mathrm{H}, \mathrm{ArH}), 7.90$ (d, 4H, J $8.7 \mathrm{~Hz}), 8.08$ (d, 4H, J $8.7 \mathrm{~Hz}) .{ }^{13} \mathrm{C} N M R(126 \mathrm{MHz}, \mathrm{DMSO}): 37.8,69.4,102.8,115.2,116.0,116.4$, 127.7, 128.2, 128.9, 129.0, 129.1, 129.2, 129.4, 130.5, 130.9, 136.0, 136.4, 136.9, 154.5, 157.9, 162.1, 162.6, 191.3. MS (m/z) $947\left(\mathrm{M}^{+}\right)$. Anal. Calcd. For $\mathrm{C}_{60} \mathrm{H}_{42} \mathrm{~N}_{4} \mathrm{O}_{4} \mathrm{~S}_{2}: \mathrm{C}, 76.09 ; \mathrm{H}, 4.47 ; \mathrm{N}, 5.92 ; \mathrm{S}, 6.77$. Found: $\mathrm{C}, 76.34 ; \mathrm{H}$, $4.33 ; \mathrm{N}, 5.69 ; \mathrm{S}, 6.44$.

\section{2,2'-(2,2'-(4,4'-(1,3-Phenylenebis(methylene))bis(oxy)bis(4,1-phenylene))bis(2-oxoethane-2,1-}

diyl))bis(sulfanediyl)bis(4,6-dimethylnicotinonitrile) (4c). Pale yellow powder, $\mathrm{mp} 171-173{ }^{\circ} \mathrm{C} ;$ yield $74 \%, \mathrm{IR}$ $\left(\mathrm{KBr}\right.$ disc) v 3062, 2916, 2215, 1680, 1598, $1421 \mathrm{~cm}^{-1} .{ }^{1} \mathrm{H}$ NMR (300 MHz, DMSO) $\delta 2.20\left(\mathrm{~s}, 6 \mathrm{H}, \mathrm{CH}_{3}\right), 2.39(\mathrm{~s}$, $\left.6 \mathrm{H}, \mathrm{CH}_{3}\right), 4.77\left(\mathrm{~s}, 4 \mathrm{H}, \mathrm{SCH}_{2}\right), 5.26\left(\mathrm{~s}, 4 \mathrm{H}, \mathrm{OCH}_{2}\right), 7.04(\mathrm{~s}, 2 \mathrm{H}$, pyridine-5-H), $7.16(\mathrm{~d}, 4 \mathrm{H}, J 9 \mathrm{~Hz}), 7.45-7.46(\mathrm{~m}, 3 \mathrm{H}$, ArH), 7.58 (s, 1H, ArH), 8.04 (d, 4H, J 8.7 Hz). ${ }^{13} \mathrm{C}$ NMR (75 MHz, DMSO): 19.5, 23.9, 36.7, 69.3, 103.5, 114.7, 115.0, 120.3, 127.0, 127.4, 128.7, 129.3, 130.6, 136.7, 152.4, 159.9, 161.1, 162.3, 191.9. MS (m/z) $698\left(\mathrm{M}^{+}\right)$. Anal. Calcd. For $\mathrm{C}_{40} \mathrm{H}_{34} \mathrm{~N}_{4} \mathrm{O}_{4} \mathrm{~S}_{2}: \mathrm{C}, 68.75 ; \mathrm{H}, 4.90 ; \mathrm{N}, 8.02 ; \mathrm{S}, 9.18$. Found: $\mathrm{C}, 68.58 ; \mathrm{H}, 4.66 ; \mathrm{N}, 8.15 ; \mathrm{S}, 9.10$. 
2,2'-(2,2'-(4,4'-(1,3-Phenylenebis(methylene))bis(oxy)bis(4,1-phenylene))bis(2-oxoethane-2,1-

diyl))bis(sulfanediyl)bis(4,6-diphenylnicotinonitrile) (4d). Pale yellow powder, $\mathrm{mp} 229-231{ }^{\circ} \mathrm{C}$; yield $77 \%$, IR $\left(\mathrm{KBr}\right.$ disc) v 3055, 2917, 2211, 1674, 1596, $1445 \mathrm{~cm}^{-1} .{ }^{1} \mathrm{H}$ NMR (300 MHz, DMSO) $\delta 4.95\left(\mathrm{~s}, 4 \mathrm{H}, \mathrm{SCH}_{2}\right), 5.32(\mathrm{~s}$, $\left.4 \mathrm{H}, \mathrm{OCH}_{2}\right), 7.12(\mathrm{~s}, 2 \mathrm{H}$, pyridine-5-H), 7.15-7.47 (m, 10H, ArH), 7.51-7.73 (m, 10H, ArH), 7.74-7.75 (m, 3H, $\operatorname{ArH}), 7.84(\mathrm{~s}, 1 \mathrm{H}, \mathrm{ArH}), 7.89$ (d, 4H, J $7.8 \mathrm{~Hz}), 8.08$ (d, 4H, J $9 \mathrm{~Hz})$. MS (m/z) $947\left(\mathrm{M}^{+}\right)$. Anal. Calcd. For $\mathrm{C}_{60} \mathrm{H}_{42} \mathrm{~N}_{4} \mathrm{O}_{4} \mathrm{~S}_{2}$ : C, 76.09; $\mathrm{H}, 4.47 ; \mathrm{N}, 5.92 ; \mathrm{S}, 6.77$. Found: C, 76.32; $\mathrm{H}, 4.28 ; \mathrm{N}, 5.77 ; \mathrm{S}, 6.57$.

Synthesis of $(4,4$ '-(phenylenebis(methylene))bis(oxy)bis(4,1-phenylene))bis((3-amino-4,6-disubstitutedthieno[2,3-b]pyridin-2-yl)methanone) 5(a-d). Method A. A mixture of the appropriate bis-bromoacetyl 2a or $\mathbf{2 b}(5 \mathrm{mmol})$ in ethanol $(25 \mathrm{~mL})$ containing sodium ethoxide $(10 \mathrm{mmol})$ and the appropriate 2-mercapto-4,6disubstituted nicotinonitrile $\mathbf{3 a}$ or $\mathbf{3 b}(10 \mathrm{mmol})$ was added. The reaction mixture was heated at reflux for $5 \mathrm{~h}$. The solid products obtained upon cooling were filtered off and recrystallized from DMF to afford the title compounds $\mathbf{5}(\mathbf{a}-\mathrm{d})$.

Method B. A solution of the appropriate 2,2'-(2,2'-(4,4'-(phenylenebis(methylene))bis(oxy)bis(4,1phenylene))bis(2-oxoethane-2,1-diyl))bis(sulfanediyl)bis(4,6-disubestitutednicotinonitrile) intermediates 4(ad) $(2 \mathrm{mmol})$ in ethanol $(25 \mathrm{~mL})$ containing sodium ethoxide $(4 \mathrm{mmol})$ was heated at reflux for $3 \mathrm{~h}$. The reaction mixture was then cooled, and the solvent was evaporated in vacuo. The solid residue was collected and recrystallized from DMF to afford $\mathbf{5}(\mathrm{a}-\mathrm{d})$.

(4,4'-(1,4-Phenylenebis(methylene))bis(oxy)bis(4,1-phenylene))bis((3-amino-4,6-dimethylthieno[2,3-

b]pyridin-2-yl)methanone) (5a). Yellow powder, mp 295-297 ${ }^{\circ} \mathrm{C}$; yield (A) $72 \%$; (B) 65\%, IR ( $\mathrm{KBr}$ disc) $v 3489$, 3437, 3070, 2972, 1593, 1554, $1462 \mathrm{~cm}^{-1} .{ }^{1} \mathrm{H}$ NMR (400 MHz, DMSO) $\delta 2.52\left(\mathrm{~s}, 6 \mathrm{H}, \mathrm{CH}_{3}\right), 2.77\left(\mathrm{~s}, 6 \mathrm{H}, \mathrm{CH}_{3}\right), 5.24$ (s, $4 \mathrm{H}, \mathrm{OCH}_{2}$ ), 7.11 (s, 2H, pyridine-5-H), $7.16(\mathrm{~d}, 4 \mathrm{H}, J 8.8 \mathrm{~Hz}), 7.54(\mathrm{~s}, 4 \mathrm{H}, \mathrm{ArH}), 7.79(\mathrm{~d}, 4 \mathrm{H}, J 8.8 \mathrm{~Hz}), 7.98(\mathrm{br}$. $\left.\mathrm{s}, 4 \mathrm{H}, \mathrm{NH}_{2}\right) .{ }^{13} \mathrm{C}$ NMR (101 MHz, DMSO): 20.5, 24.4, 69.6, 102.8, 114.9, 122.1, 122.4, 128.4, 130.0, 130.9, 134.0, 136.8, 146.3, 152.8, 161.0, 161.8, 188.4. MS (m/z) $698\left(\mathrm{M}^{+}\right)$. Anal. Calcd. For $\mathrm{C}_{40} \mathrm{H}_{34} \mathrm{~N}_{4} \mathrm{O}_{4} \mathrm{~S}_{2}: \mathrm{C}, 68.75 ; \mathrm{H}, 4.90 ; \mathrm{N}$, 8.02; S, 9.18. Found: C, 68.49; H, 4.61; N, 8.24; S, 9.32.

(4,4'-(1,4-Phenylenebis(methylene))bis(oxy)bis(4,1-phenylene))bis((3-amino-4,6-diphenylthieno[2,3-

b]pyridin-2-yl)methanone) (5b). Yellow powder, mp 263-265 ${ }^{\circ} \mathrm{C}$; yield (A) 73\%; (B) 70\%, IR ( $\mathrm{KBr}$ disc) v 3466, $3257,2918,1593,1543,1432 \mathrm{~cm}^{-1}$. ${ }^{1} \mathrm{H}$ NMR (300 MHz, DMSO) $\delta 5.25$ (s, 4H, OCH $)_{2}, 6.84$ (br. s, 4H, NH $), 7.18$ (d, 4H, J 8.7 Hz), 7.50-7.63 (m, 20H, ArH), $7.82(\mathrm{~s}, 2 \mathrm{H}$, pyridine-5-H), $7.83(\mathrm{~d}, 4 \mathrm{H}, J 8.7 \mathrm{~Hz}), 8.22-8.25(\mathrm{~m}, 4 \mathrm{H}$, ArH). ${ }^{13} \mathrm{C}$ NMR (101 MHz, DMSO): 69.6, 103.9, 113.4, 115.0, 118.8, 120.3, 127.7, 128.4, 128.9, 129.4, 129.5, 130.1, 130.9, 133.8, 136.6, 136.9, 137.6, 149.2, 150.2, 157.6, 161.2, 162.4, 188.4. MS (m/z) 947 (M ${ }^{+}$. Anal. Calcd. For $\mathrm{C}_{60} \mathrm{H}_{42} \mathrm{~N}_{4} \mathrm{O}_{4} \mathrm{~S}_{2}$ : C, 76.09; $\mathrm{H}, 4.47 ; \mathrm{N}, 5.92 ; \mathrm{S}, 6.77$. Found: $\mathrm{C}, 76.23 ; \mathrm{H}, 4.26 ; \mathrm{N}, 5.80 ; \mathrm{S}, 6.60$.

(4,4'-(1,3-Phenylenebis(methylene))bis(oxy)bis(4,1-phenylene))bis((3-amino-4,6-dimethylthieno[2,3-

b]pyridin-2-yl)methanone) (5c). Yellow powder, mp 230-231 ${ }^{\circ} \mathrm{C}$; yield (A) 72\%; (B) 67\%, IR (KBr disc) $v 3480$, 3436, 3038, 2972, 1593, 1552, $1463 \mathrm{~cm}^{-1} .{ }^{1} \mathrm{H}$ NMR (300 MHz, DMSO) $\delta 2.48\left(\mathrm{~s}, 6 \mathrm{H}, \mathrm{CH}_{3}\right), 2.76\left(\mathrm{~s}, 6 \mathrm{H}, \mathrm{CH}_{3}\right), 5.24$ $\left(\mathrm{s}, 4 \mathrm{H}, \mathrm{OCH}_{2}\right), 7.08(\mathrm{~s}, 2 \mathrm{H}$, pyridine-5-H), $7.15(\mathrm{~d}, 4 \mathrm{H}, \mathrm{J} 8.7 \mathrm{~Hz}), 7.47(\mathrm{~s}, 3 \mathrm{H}, \mathrm{ArH}), 7.61(\mathrm{~s}, 1 \mathrm{H}, \mathrm{ArH}), 7.78(\mathrm{~d}, 4 \mathrm{H}, J$ $8.4 \mathrm{~Hz}$ ), 7.94 (br. s, 4H, NH${ }_{2}$ ). ${ }^{13} \mathrm{C}$ NMR (75 MHz, DMSO): 20.1, 24.0, 69.3, 102.4, 114.5, 120.6, 121.6, 121.9, 127.1, 127.4, 128.7, 129.5, 133.6, 137.0, 145.8, 152.3, 160.5, 161.3, 187.9. MS (m/z) $698\left(\mathrm{M}^{+}\right)$. Anal. Calcd. For $\mathrm{C}_{40} \mathrm{H}_{34} \mathrm{~N}_{4} \mathrm{O}_{4} \mathrm{~S}_{2}: \mathrm{C}, 68.75 ; \mathrm{H}, 4.90 ; \mathrm{N}, 8.02 ; \mathrm{S}, 9.18$. Found: C, 68.57; H, 4.77; N, 8.19; S, 9.08.

(4,4'-(1,3-Phenylenebis(methylene))bis(oxy)bis(4,1-phenylene))bis((3-amino-4,6-diphenylthieno[2,3-

b]pyridin-2-yl)methanone) (5d). Yellow powder, mp 241-243 ${ }^{\circ} \mathrm{C}$; yield (A) 77\%; (B) 68\%, IR ( $\mathrm{KBr}$ disc) v 3469, 3257, 2955, 1603, 1543, $1462 \mathrm{~cm}^{-1} .{ }^{1} \mathrm{H}$ NMR (300 MHz, DMSO) $\delta 5.27$ (s, 4H, OCH $), 6.63$ (d, $4 \mathrm{H}, J 7.5 \mathrm{~Hz}$ ), 6.84 (br. s, 4H, NH $\mathrm{NH}_{2}, 6.95$ (d, 4H, J $\left.8.7 \mathrm{~Hz}\right), 7.18(\mathrm{~d}, 2 \mathrm{H}, J 8.1 \mathrm{~Hz}), 7.48-7.63(\mathrm{~m}, 14 \mathrm{H}, \mathrm{ArH}), 7.81(\mathrm{~s}, 1 \mathrm{H}, \mathrm{ArH}), 7.84(\mathrm{~s}$, $2 \mathrm{H}$, pyridine-5-H), 8.21-8.51 (m, 7H, ArH). MS (m/z) $947\left(\mathrm{M}^{+}\right)$. Anal. Calcd. For $\mathrm{C}_{60} \mathrm{H}_{42} \mathrm{~N}_{4} \mathrm{O}_{4} \mathrm{~S}_{2}$ : C, 76.09; $\mathrm{H}, 4.47$; N, 5.92; S, 6.77. Found: C, 75.93; H, 4.31; N, 5.76; S, 6.88. 
Synthesis of 1,1'-(4,4'-(pyridine-2,6-diylbis(methylene))bis(oxy)bis(4,1-phenylene))diethanone (8). A solution of potassium 4-acetylphenolate $(20 \mathrm{mmol}$ ) and 2,6-bis(bromo-methyl)pyridine (7) (10 mmol) in DMF (20 mL) was heated under reflux for 15 min., during which time, $\mathrm{KBr}$ precipitated. The solvent was then removed in vacuo, and the remaining material was washed with water $(50 \mathrm{~mL})$ and purified by crystallization from ethanol.

1,1'-(4,4'-(Pyridine-2,6-diylbis(methylene))bis(oxy)bis(4,1-phenylene))diethanone (8). Pale yellow crystals, $\mathrm{mp} 145-148^{\circ} \mathrm{C}$; yield 78\%, IR (KBr disc) v 3073, 2962, 1673, 1598, $1471 \mathrm{~cm}^{-1} .{ }^{1} \mathrm{H}$ NMR (300 MHz, DMSO) $\delta 2.51$ $\left(\mathrm{s}, 6 \mathrm{H}, \mathrm{CH}_{3}\right), 5.30\left(\mathrm{~s}, 4 \mathrm{H}, \mathrm{OCH}_{2}\right), 7.14(\mathrm{~d}, 4 \mathrm{H}, J 9.0 \mathrm{~Hz}), 7.49(\mathrm{~d}, 2 \mathrm{H}, J 7.8 \mathrm{~Hz}), 7.89(\mathrm{t}, 1 \mathrm{H}, J 7.8 \mathrm{~Hz}), 7.93(\mathrm{~d}, 4 \mathrm{H}, J$ 9.0). MS (m/z) $375\left(\mathrm{M}^{+}\right)$. Anal. Calcd. For $\mathrm{C}_{23} \mathrm{H}_{21} \mathrm{NO}_{4}$ : C, 73.58; $\mathrm{H}, 5.64 ; \mathrm{N}, 3.73$. Found: $\mathrm{C}, 73.36 ; \mathrm{H}, 5.49 ; \mathrm{N}$, 3.65 .

Synthesis of 1,1'-(4,4'-(pyridine-2,6-diylbis(methylene))bis(oxy)bis(4,1-phenylene))bis(2-bromoethanone) (9). To a stirred solution of 1,1'-(4,4'-(pyridine-2,6-diylbis(methylene))bis(oxy)bis(4,1-phenylene))diethanone (8) $(10 \mathrm{mmol})$ and $p$-TsOH $(20 \mathrm{mmol})$ in acetonitrile $(50 \mathrm{~mL})$, NBS $(20 \mathrm{mmol})$ was slowly added. After complete addition of NBS, the reaction mixture was heated at reflux with stirring for $5 \mathrm{~h}$. The solvent was then evaporated in vacuo and the residue was stirred with water $(50 \mathrm{~mL})$ for $1 \mathrm{~h}$. and then filtered. After filtration the resulting solid was recrystallized from acetone to afford compound 9.

1,1'-(4,4'-(Pyridine-2,6-diylbis(methylene))bis(oxy)bis(4,1-phenylene))bis(2-bromoethanone) (9). White crystals, mp $116-118{ }^{\circ} \mathrm{C}$; yield $72 \%$, IR ( $\mathrm{KBr}$ disc) v 3069, 2992, 1694, 1597, $1445 \mathrm{~cm}^{-1} .{ }^{1} \mathrm{H} \mathrm{NMR}(300 \mathrm{MHz}$, $\left.\mathrm{CDCl}_{3}\right) \delta 4.40\left(\mathrm{~s}, 4 \mathrm{H}, \mathrm{CH}_{2} \mathrm{Br}\right), 5.35\left(\mathrm{~s}, 4 \mathrm{H}, \mathrm{OCH}_{2}\right), 7.07(\mathrm{~d}, 4 \mathrm{H}, J 8.7 \mathrm{~Hz}), 7.53(\mathrm{~d}, 2 \mathrm{H}, J 7.8 \mathrm{~Hz}), 7.86(\mathrm{t}, 1 \mathrm{H}, J 7.8 \mathrm{~Hz})$, 7.99 (d, 4H, J 8.7). MS (m/z) $533\left(\mathrm{M}^{+}\right)$. Anal. Calcd. For $\mathrm{C}_{23} \mathrm{H}_{19} \mathrm{Br}_{2} \mathrm{NO}_{4}$ : C, 51.81; H, 3.59; $\mathrm{Br}, 29.97 ; \mathrm{N}, 2.63$. Found: C, 51.66; $\mathrm{H}, 3.40 ; \mathrm{Br}, 29.81 ; \mathrm{N}, 2.47$.

Synthesis of 2,2'-(2,2'-(4,4'-(pyridine-2,6-diylbis(methylene))bis(oxy)bis(4,1-phenylene))bis(2-oxoethane-2,1diyl))bis(sulfanediyl)bis(4,6-disubstituted nicotinonitrile) $\mathbf{1 0 ( a - b ) . ~ T o ~ a ~ s o l u t i o n ~ o f ~ 1 , 1 ' - ( 4 , 4 ' - ( p y r i d i n e - 2 , 6 - ~}$ diylbis(methylene))bis(oxy)bis(4,1-phenylene))bis(2-bromoethanone) (9) (5 mmol) in ethanol (25 mL) containing few drops piperidine, the appropriate 2-mercapto-4,6-disubstituted nicotinonitriles $\mathbf{3 a}$ or $\mathbf{3 b}$ (10 $\mathrm{mmol}$ ) was added. The reaction mixture was heated at reflux for $2 \mathrm{~h}$. The solid product obtained upon cooling was filtered off and recrystallized from ethanol/ DMF to afford the title compounds 10(a-b).

2,2'-(2,2'-(4,4'-(Pyridine-2,6-diylbis(methylene))bis(oxy)bis(4,1-phenylene))bis(2-oxoethane-2,1-diyl))bis(sulfanediyl)bis(4,6-dimethylnicotinonitrile) (10a). Pale yellow powder, $\mathrm{mp} 179-181{ }^{\circ} \mathrm{C}$; yield $73 \%, \mathrm{IR}(\mathrm{KBr}$ disc) v 3065, 2964, 2216, 1668, 1594, $1437 \mathrm{~cm}^{-1} .{ }^{1} \mathrm{H}$ NMR (300 MHz, DMSO) $\delta 2.19\left(\mathrm{~s}, 6 \mathrm{H}, \mathrm{CH}_{3}\right), 2.39(\mathrm{~s}, 6 \mathrm{H}$, $\left.\mathrm{CH}_{3}\right), 4.76\left(\mathrm{~s}, 4 \mathrm{H}, \mathrm{SCH}_{2}\right), 5.33\left(\mathrm{~s}, 4 \mathrm{H}, \mathrm{OCH}_{2}\right), 7.03(\mathrm{~s}, 2 \mathrm{H}$, pyridine-5-H), $7.19(\mathrm{~d}, 4 \mathrm{H}, J 6.9 \mathrm{~Hz}), 7.51(\mathrm{~d}, 2 \mathrm{H}, J 8.1$ $\mathrm{Hz}) 7.91(\mathrm{t}, 1 \mathrm{H}, J 7.5 \mathrm{~Hz}), 8.05$ (d, $4 \mathrm{H}, J 7.2 \mathrm{~Hz})$. MS (m/z) $699\left(\mathrm{M}^{+}\right)$. Anal. Calcd. For $\mathrm{C}_{39} \mathrm{H}_{33} \mathrm{~N}_{5} \mathrm{O}_{4} \mathrm{~S}_{2}: \mathrm{C}, 66.93 ; \mathrm{H}$, 4.75; N, 10.01; S, 9.16. Found: C, 66.82; H, 4.67; N, 10.15; S, 9.09.

2,2'-(2,2'-(4,4'-(Pyridine-2,6-diylbis(methylene))bis(oxy)bis(4,1-phenylene))bis(2-oxoethane-2,1-diyl))bis(sulfanediyl)bis(4,6-diphenylnicotinonitrile) (10b). Pale yellow powder, $\mathrm{mp} 198-200{ }^{\circ} \mathrm{C}$; yield $75 \%, \mathrm{IR}(\mathrm{KBr}$ disc) v 3056, 2897, 2211, 1674, 1596, $1451 \mathrm{~cm}^{-1} .{ }^{1} \mathrm{H}$ NMR $(300 \mathrm{MHz}, \mathrm{DMSO}) \delta 4.96(\mathrm{~s}, 4 \mathrm{H}, \mathrm{SCH}), 5.39(\mathrm{~s}, 4 \mathrm{H}$, $\mathrm{OCH}_{2}$ ), $7.12(\mathrm{~s}, 2 \mathrm{H}$, pyridine-5-H), $7.16(\mathrm{~d}, 2 \mathrm{H}, J 8.1 \mathrm{~Hz}), 7.21(\mathrm{~d}, 4 \mathrm{H}, J 8.7 \mathrm{~Hz}), 7.36(\mathrm{t}, 1 \mathrm{H}, J 7.2 \mathrm{~Hz}), 7.52-7.9(\mathrm{~m}$, $20 \mathrm{H}, \mathrm{ArH}), 8.11$ (d, 4H, J $9 \mathrm{~Hz}) .{ }^{13} \mathrm{C}$ NMR (75 MHz, DMSO): 37.6, 70.4, 102.5, 113.1, 114.9, 115.7, 116.1, 118.3, $121.1,127.3,128.5,129.2,130.5,131.5,135.5,136.1,138.1,154.1,155.9,157.8,161.9,162.2,191.2$. MS $(\mathrm{m} / \mathrm{z}) 948\left(\mathrm{M}^{+}\right)$. Anal. Calcd. For $\mathrm{C}_{59} \mathrm{H}_{41} \mathrm{~N}_{5} \mathrm{O}_{4} \mathrm{~S}_{2}: \mathrm{C}, 74.74 ; \mathrm{H}, 4.36 ; \mathrm{N}, 7.39 ; \mathrm{S}, 6.76$. Found: $\mathrm{C}, 74.56 ; \mathrm{H}, 4.29 ; \mathrm{N}$, $7.21 ; \mathrm{S}, 6.67$.

Synthesis of $\quad\left(4,4^{\prime}-(p y r i d i n e-2,6-d i y l b i s(m e t h y l e n e)) b i s(o x y) b i s(4,1-p h e n y l e n e)\right) b i s((3-a m i n o-4,6-$ disubstituted thieno[2,3-b]pyridin-2-yl)methanone)) 11(a-b). Method A: A mixture of 1,1'-(4,4'-(pyridine-2,6diylbis(methylene))bis(oxy)bis(4,1-phenylene))bis(2-bromoethanone) (9) (5 mmol) in ethanol (25 $\mathrm{mL})$ 
containing sodium ethoxide (10 $\mathrm{mmol})$ and the appropriate 2-mercapto-4,6-disubstituted nicotinonitrile 3a or $\mathbf{3 b}(10 \mathrm{mmol})$ was added. The reaction mixture was heated at reflux for $5 \mathrm{~h}$. The solid products obtained upon cooling were filtered off and recrystallized from DMF to afford the title compounds 11(a-b).

Method B. A solution of 2,2'-(2,2'-(4,4'-(pyridine-2,6-diylbis(methylene))bis(oxy)bis-(4,1-phenylene))bis(2oxoethane-2,1-diyl))bis(sulfanediyl)bis(4,6-disubstituted-nicotinonitrile) intermediates 10a or 10b (2 mmol) in ethanol $(25 \mathrm{~mL})$ containing sodium ethoxide $(4 \mathrm{mmol})$ was heated at reflux for $2 \mathrm{~h}$. The reaction mixture was then cooled, and the solvent was evaporated in vacuo. The solid residue was collected and recrystallized from DMF to afford 11(a-b).

(4,4'-(Pyridine-2,6-diylbis(methylene))bis(oxy)bis(4,1-phenylene))bis((3-amino-4,6-dimethylthieno[2,3b]pyridin-2-yl)methanone) (11a). Yellow powder, mp 276-278 ${ }^{\circ} \mathrm{C}$; yield (A) 70\%; (B) 68\%, IR ( $\mathrm{KBr}$ disc) v 3496, 3306, 3069, 2976, 1593, 1556, $1440 \mathrm{~cm}^{-1} .{ }^{1} \mathrm{H}$ NMR (300 MHz, DMSO) $\delta 2.48\left(\mathrm{~s}, 6 \mathrm{H}, \mathrm{CH}_{3}\right), 2.75\left(\mathrm{~s}, 6 \mathrm{H}, \mathrm{CH}_{3}\right), 5.31$ $\left(\mathrm{s}, 4 \mathrm{H}, \mathrm{OCH}_{2}\right), 7.07$ (s, 2H, pyridine-5-H), $7.18(\mathrm{~d}, 4 \mathrm{H}, J 8.4 \mathrm{~Hz}), 7.54(\mathrm{~d}, 2 \mathrm{H}, J 7.8 \mathrm{~Hz}), 7.79(\mathrm{~d}, 4 \mathrm{H}, J 8.7 \mathrm{~Hz}), 7.93-$ $7.94\left(\mathrm{~m}, 5 \mathrm{H}, \mathrm{ArH} \& \mathrm{NH}_{2}\right)$. MS $(\mathrm{m} / z) 699\left(\mathrm{M}^{+}\right)$. Anal. Calcd. For $\mathrm{C}_{39} \mathrm{H}_{33} \mathrm{~N}_{5} \mathrm{O}_{4} \mathrm{~S} 2$ : C, 66.93; H, 4.75; N, 10.01; S, 9.16. Found: $\mathrm{C}, 66.72 ; \mathrm{H}, 4.57 ; \mathrm{N}, 10.19 ; \mathrm{S}, 9.00$.

(4,4'-(Pyridine-2,6-diylbis(methylene))bis(oxy)bis(4,1-phenylene))bis((3-amino-4,6-diphenylthieno[2,3b]pyridin-2-yl)methanone) (11b). Yellow powder, mp 258-260 ${ }^{\circ} \mathrm{C}$; yield (A) 71\%; (B) 67\%, IR (KBr disc) v 3467, 3257, 3055, 2919, 1590, 1533, $1434 \mathrm{~cm}^{-1} .{ }^{1} \mathrm{H}$ NMR (300 MHz, DMSO) $\delta 5.34\left(\mathrm{~s}, 4 \mathrm{H}, \mathrm{OCH}_{2}\right), 6.83\left(\mathrm{br} . \mathrm{s}, 4 \mathrm{H}, \mathrm{NH}_{2}\right)$, $7.22(\mathrm{~d}, 4 \mathrm{H}, J 9.0 \mathrm{~Hz}), 7.50-7.53(\mathrm{~m}, 8 \mathrm{H}, \mathrm{ArH}), 7.56(\mathrm{~d}, 2 \mathrm{H}, J 7.5 \mathrm{~Hz}), 7.63-7.85(\mathrm{~m}, 12 \mathrm{H}, \operatorname{ArH}), 7.86(\mathrm{~s}, 2 \mathrm{H}$, pyridine-5-H), $7.95(t, 1 \mathrm{H}, J 7.5 \mathrm{~Hz}), 8.23(\mathrm{~d}, 4 \mathrm{H}, J 7.8 \mathrm{~Hz}), \mathrm{MS}(\mathrm{m} / \mathrm{z}) 948\left(\mathrm{M}^{+}\right)$. Anal. Calcd. For $\mathrm{C}_{59} \mathrm{H}_{41} \mathrm{~N}_{5} \mathrm{O}_{4} \mathrm{~S}_{2}: \mathrm{C}_{\text {, }}$ 74.74; H, 4.36; N, 7.39; S, 6.76. Found: C, 74.63; H, 4.13; N, 7.66; S, 6.69.

Synthesis of diethyl 3,4-bis((4-(2-(3-cyano-4,6-disubstituted pyridin-2-ylthio)acetyl)phenoxy)methyl)thieno-

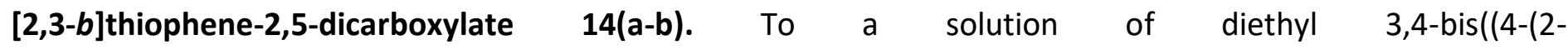
bromoacetyl)phenoxy)methyl)thieno[2,3-b]thiophene-2,5-dicarboxylate (13) (5 mmol) in ethanol (25 $\mathrm{mL})$ containing few drops piperidine, the appropriate 2-mercapto-4,6-disubstituted nicotinonitriles $\mathbf{3 a}$ or $\mathbf{3 b}$ (10 $\mathrm{mmol}$ ) was added. The reaction mixture was heated at reflux for $2 \mathrm{~h}$. The solid product obtained upon cooling was filtered off and recrystallized from ethanol/ DMF to afford the title compounds 14(a-b).

Diethyl 3,4-bis((4-(2-(3-cyano-4,6-dimethylpyridin-2-ylthio)acetyl)phenoxy)methyl)thieno[2,3-b]thiophene-

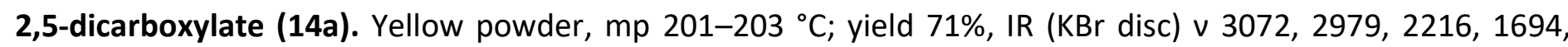
1600, 1578, $1448 \mathrm{~cm}^{-1} .{ }^{1} \mathrm{H}$ NMR (300 MHz, DMSO) $\delta 1.27(\mathrm{t}, 6 \mathrm{H}, J 6.9 \mathrm{~Hz}), 2.16\left(\mathrm{~s}, 6 \mathrm{H}, \mathrm{CH}_{3}\right), 2.36\left(\mathrm{~s}, 6 \mathrm{H}, \mathrm{CH}_{3}\right)$, $4.33(\mathrm{q}, 4 \mathrm{H}, J 6.9 \mathrm{~Hz}), 4.65\left(\mathrm{~s}, 4 \mathrm{H}, \mathrm{SCH}_{2}\right), 5.70\left(\mathrm{~s}, 4 \mathrm{H}, \mathrm{OCH}_{2}\right), 6.97(\mathrm{~s}, 2 \mathrm{H}$, pyridine-5-H), $6.99(\mathrm{~d}, 4 \mathrm{H}, J 8.4 \mathrm{~Hz})$, $7.87(\mathrm{~d}, 4 \mathrm{H}, J 8.4 \mathrm{~Hz})$. MS (m/z) $905\left(\mathrm{M}^{+}\right)$. Anal. Calcd. For $\mathrm{C}_{46} \mathrm{H}_{40} \mathrm{~N}_{4} \mathrm{O}_{8} \mathrm{~S}_{4}: \mathrm{C}, 61.04 ; \mathrm{H}, 4.45 ; \mathrm{N}, 6.19 ; \mathrm{S}, 14.17$. Found: C, 61.15; H, 4.29; N, 6.02; S, 14.25.

Diethyl 3,4-bis((4-(2-(3-cyano-4,6-diphenylpyridin-2-ylthio)acetyl)phenoxy)methyl)thieno[2,3-b]thiophene2,5-dicarboxylate (14b). Yellow powder, mp 215-218 ${ }^{\circ} \mathrm{C}$; yield 76\%, IR ( $\mathrm{KBr}$ disc) v 3057, 2976, 2214, 1706, 1676, 1598, $1447 \mathrm{~cm}^{-1} .{ }^{1} \mathrm{H}$ NMR (400 MHz, DMSO) $\delta 1.31(\mathrm{t}, 6 \mathrm{H}, J 7.2 \mathrm{~Hz}$ ), 4.37 (q, 4H, J 7.2 Hz ), $4.63(\mathrm{~s}, 4 \mathrm{H}$, $\left.\mathrm{SCH}_{2}\right), 5.70\left(\mathrm{~s}, 4 \mathrm{H}, \mathrm{OCH}_{2}\right), 6.90(\mathrm{~d}, 4 \mathrm{H}, J 8.8 \mathrm{~Hz}), 7.16-7.72(\mathrm{~m}, 16 \mathrm{H}, \mathrm{ArH}), 7.75(\mathrm{~d}, 4 \mathrm{H}, J 8.8 \mathrm{~Hz}), 7.81(\mathrm{~s}, 2 \mathrm{H}$, pyridine-5-H), 7.85-7.87 (m, 4H, ArH). ${ }^{13} \mathrm{C} N M R(126 \mathrm{MHz}, \mathrm{DMSO}): 14.4,37.8,40.5,62.2,102.9,114.6,116.0$, 116.6, 127.9, 129.0, 129.06, 129.1, 129.2, 129.4, 130.5, 130.9, 135.5, 135.9, 136.8, 145.8, 146.1, 154.5, 158.3, 161.7, 162.1, 162.3, 191.3. Anal. Calcd. For $\mathrm{C}_{66} \mathrm{H}_{48} \mathrm{~N}_{4} \mathrm{O}_{8} \mathrm{~S}_{4}: \mathrm{C}, 68.73 ; \mathrm{H}, 4.19 ; \mathrm{N}, 4.86 ; \mathrm{S}, 11.12$. Found: C, 68.59; $\mathrm{H}, 4.11 ; \mathrm{N}, 4.70 ; \mathrm{S}, 11.21$.

Synthesis of sodium 3,4-bis((4-(3-amino-4,6-disubstitutedthieno[2,3-b]-pyridine-2-carbonyl)phenoxy)methyl)thieno[2,3-b]thiophene-2,5-dicarboxylate 15(a-b). Method A. A mixture of diethyl 3,4-bis((4-(2bromoacetyl)phenoxy)methyl)thieno-[2,3-b]thiophene-2,5-dicarboxylate (13) (5 mmol) in ethanol (25 $\mathrm{mL})$ containing sodium ethoxide $(10 \mathrm{mmol})$ and the appropriate 2-mercapto-4,6-disubstituted nicotinonitrile 3a or 
$3 \mathbf{b}(10 \mathrm{mmol})$ was added. The reaction mixture was heated at reflux for $5 \mathrm{~h}$. The solid products obtained upon cooling were filtered off and recrystallized from DMF to afford the title compounds 15(a-b).

Method B. A solution of diethyl 3,4-bis((4-(2-(3-cyano-4,6-disubstitutedpyridin-2-ylthio)acetyl)phenoxy) methyl)thieno[2,3-b]thiophene-2,5-dicarboxylate $14 \mathbf{a}$ or $14 \mathbf{b}(2 \mathrm{mmol})$ in ethanol $(25 \mathrm{~mL})$ containing sodium ethoxide $(4 \mathrm{mmol}$ ) was heated at reflux for $2 \mathrm{~h}$. The reaction mixture was then cooled, and the solvent was evaporated in vacuo. The solid residue was collected and recrystallized from DMF to afford 15(a-b).

Sodium 3,4-bis((4-(3-amino-4,6-dimethylthieno[2,3-b]pyridine-2-carbonyl)phenoxy)methyl)thieno[2,3b]thiophene-2,5-dicarboxylate (15a). Yellow powder, $\mathrm{mp}>300^{\circ} \mathrm{C}$; yield (A) 69\%; (B) 67\%, IR (KBr disc) v 3431, 3330, 2965, 1599, 1550, 1502, $1442 \mathrm{~cm}^{-1} .{ }^{1} \mathrm{H}$ NMR (500 MHz, DMSO) $2.47\left(\mathrm{~s}, 6 \mathrm{H}, \mathrm{CH}_{3}\right), 2.72\left(\mathrm{~s}, 6 \mathrm{H}, \mathrm{CH}_{3}\right), 5.99$ (s, $4 \mathrm{H}, \mathrm{OCH}_{2}$ ), $7.00\left(\mathrm{~s}, 2 \mathrm{H}\right.$, pyridine-5-H), $7.13(\mathrm{~d}, 4 \mathrm{H}, J 8.5 \mathrm{~Hz}), 7.65(\mathrm{~d}, 4 \mathrm{H}, J 8.5 \mathrm{~Hz}), 7.84\left(\mathrm{br} . \mathrm{s}, 4 \mathrm{H}, \mathrm{NH}_{2}\right) .{ }^{13} \mathrm{C}$ NMR (126 MHz, DMSO): 20.5, 24.3, 61.7, 102.9, 114.6, 121.9, 122.1, 129.2, 129.9, 133.2, 137.2, 145.9, 148.0, 149.3, 152.5, 160.6, 161.0, 161.6, 165.2, 188.2. MS (m/z) $892\left(\mathrm{M}^{+}\right)$. Anal. Calcd. For $\mathrm{C}_{42} \mathrm{H}_{30} \mathrm{~N}_{4} \mathrm{Na}_{2} \mathrm{O}_{8} \mathrm{~S}_{4}: \mathrm{C}, 56.49$; $H, 3.39 ; \mathrm{N}, 6.27 ; \mathrm{S}, 14.36$. Found: C, 56.32; H, 3.25; N, 6.11; S, 14.31 .

Sodium 3,4-bis((4-(3-amino-4,6-diphenylthieno[2,3-b]pyridine-2-carbonyl)phenoxy)methyl)thieno[2,3b]thiophene-2,5-dicarboxylate (15b). Yellow powder, $\mathrm{mp}>300^{\circ} \mathrm{C}$; yield (A) 72\%; (B) 69\%, IR (KBr disc) v 3469, $3277,2919,1583,1534,1500,1440 \mathrm{~cm}^{-1} .{ }^{1} \mathrm{H}$ NMR (400 MHz, DMSO) $\delta 6.03\left(\mathrm{~s}, 4 \mathrm{H}, \mathrm{OCH}_{2}\right), 6.73\left(\mathrm{br} . \mathrm{s}, 4 \mathrm{H}, \mathrm{NH}_{2}\right)$, $7.19(\mathrm{~d}, 4 \mathrm{H}, \mathrm{J} 8.8 \mathrm{~Hz}), 7.47-7.71(\mathrm{~m}, 2 \mathrm{H}, \mathrm{ArH}), 7.73\left(\mathrm{~s}, 2 \mathrm{H}\right.$, pyridine-5-H), 8.17-8.32 (m, 4H, ArH). ${ }^{13} \mathrm{C} \mathrm{NMR}(126$ MHz, DMSO): 61.8, 104.1, 114.7, 118.5, 120.2, 127.6, 128.9, 129.1, 129.2, 129.4, 129.8, 130.0, 130.4, 133.0, 136.6, 137.3, 137.6, 148.0, 148.8, 149.3, 149.9, 157.2, 161.3, 162.3, 165.2, 188.3. Anal. Calcd. For $\mathrm{C}_{62} \mathrm{H}_{38} \mathrm{~N}_{4} \mathrm{Na}_{2} \mathrm{O}_{8} \mathrm{~S}_{4}:$ C, 65.25; $\mathrm{H}, 3.36 ; \mathrm{N}, 4.91 ; \mathrm{S}, 11.24$. Found: $\mathrm{C}, 65.03 ; \mathrm{H}, 3.16 ; \mathrm{N}, 4.73 ; \mathrm{S}, 11.14$.

Synthesis of 3,4-bis((4-(3-amino-4,6-diphenylthieno[2,3-b]pyridine-2-carbonyl)phenoxy)methyl)thieno[2,3b]thiophene-2,5-dicarboxylic acid (16). To a stirred a solution of sodium 3,4-bis((4-(3-amino-4,6diphenylthieno[2,3-b]-pyridine-2-carbonyl)phenoxy)methyl)thieno[2,3-b]thiophene-2,5-dicarboxylate (15b) (2 $\mathrm{mmol}$ ) in $25 \mathrm{ml}$ water, few drops of conc. $\mathrm{HCl}$ were add with stirring. After $1 \mathrm{~h}$. the obtained solid product was filtrated to give compound 16.

3,4-Bis((4-(3-amino-4,6-diphenylthieno[2,3-b]pyridine-2-carbonyl)phenoxy)methyl)thieno[2,3-b]thiophene-

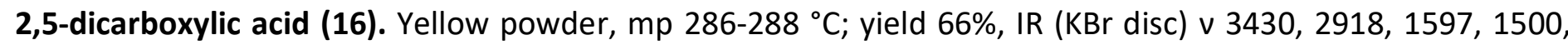
$1434 \mathrm{~cm}^{-1}$. ${ }^{1} \mathrm{H}$ NMR (300 MHz, DMSO) $\delta 3.4$ (br. s, 2H, OH), 5.72 (s, 4H, OCH $), 6.72$ (br. s, 4H, NH ), 6.99 (d, 4H, J $8.4 \mathrm{~Hz}), 7.41-7.67(\mathrm{~m}, 20 \mathrm{H}, \mathrm{ArH}), 7.70(\mathrm{~s}, 2 \mathrm{H}$, pyridine-5-H), 8.08-8.11 (m, 4H, ArH). Anal. Calcd. For $\mathrm{C}_{62} \mathrm{H}_{40} \mathrm{~N}_{4} \mathrm{O}_{8} \mathrm{~S}_{4}: \mathrm{C}, 67.87 ; \mathrm{H}, 3.67 ; \mathrm{N}, 5.11 ; \mathrm{S}, 11.69$. Found: $\mathrm{C}, 67.76 ; \mathrm{H}, 3.59 ; \mathrm{N}, 4.98 ; \mathrm{S}, 11.49$.

Synthesis of diethyl 3,4-bis((2-(2-(3-cyano-4,6-disubstitutedpyridin-2-ylthio)acetyl)phenoxy)methyl)thieno[2,3-b]thiophene-2,5-dicarboxylate 19(a-b). To a solution of diethyl 3,4-bis((2-(2bromoacetyl)phenoxy)methyl)thieno[2,3-b]-thiophene-2,5-dicarboxylate (18) (5 mmol) in ethanol (25 $\mathrm{mL})$ containing few drops piperidine, the appropriate 2-mercapto-4,6-disubstituted nicotinonitriles $\mathbf{3 a}$ or $\mathbf{3 b}$ (10 $\mathrm{mmol}$ ) was added. The reaction mixture was heated at reflux for $2 \mathrm{~h}$. The solid product obtained upon cooling was filtered off and recrystallized from ethanol/ DMF to afford the title compounds 19(a-b).

Diethyl 3,4-bis((2-(2-(3-cyano-4,6-dimethylpyridin-2-ylthio)acetyl)phenoxy)methyl)thieno[2,3-b]thiophene2,5-dicarboxylate (19a). Pale yellow powder, $\mathrm{mp} 116-118{ }^{\circ} \mathrm{C}$; yield $71 \%$, IR ( $\mathrm{KBr}$ disc) v 3066, 2979, 2216, 1709, 1594, 1580, $1447 \mathrm{~cm}^{-1} .{ }^{1} \mathrm{H}$ NMR (400 MHz, DMSO) $\delta 1.21\left(\mathrm{t}, 6 \mathrm{H}, J 6.8 \mathrm{~Hz}\right.$ ), $2.06\left(\mathrm{~s}, 6 \mathrm{H}, \mathrm{CH}_{3}\right), 2.35(\mathrm{~s}, 6 \mathrm{H}$, $\left.\mathrm{CH}_{3}\right), 4.26(\mathrm{q}, 4 \mathrm{H}, \mathrm{J} 6.8 \mathrm{~Hz}), 4.33\left(\mathrm{~s}, 4 \mathrm{H}, \mathrm{SCH}_{2}\right), 5.67\left(\mathrm{~s}, 4 \mathrm{H}, \mathrm{OCH}_{2}\right), 6.94(\mathrm{~s}, 2 \mathrm{H}$, pyridine-5-H), 6.95-7.59 (m, 8H, ArH). MS (m/z) $905\left(\mathrm{M}^{+}\right)$. Anal. Calcd. For $\mathrm{C}_{46} \mathrm{H}_{40} \mathrm{~N}_{4} \mathrm{O}_{8} \mathrm{~S}_{4}: \mathrm{C}, 61.04 ; \mathrm{H}, 4.45 ; \mathrm{N}, 6.19 ; \mathrm{S}, 14.17$. Found: $\mathrm{C}, 60.81 ; \mathrm{H}$, 4.29; N, 6.10; S, 14.05.

Diethyl 3,4-bis((2-(2-(3-cyano-4,6-diphenylpyridin-2-ylthio)acetyl)phenoxy)methyl)thieno[2,3-b]thiophene-

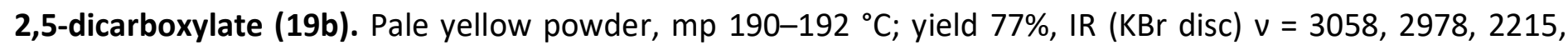


1706, 1595, 1569, $1446 \mathrm{~cm}^{-1} .{ }^{1} \mathrm{H}$ NMR (300 MHz, DMSO) $\delta 1.05$ (t, 6H, J 7.2 Hz), 4.06 (q, 4H, J 7.2 Hz), 4.51 (s, 4H, $\left.\mathrm{SCH}_{2}\right), 5.62\left(\mathrm{~s}, 4 \mathrm{H}, \mathrm{OCH}_{2}\right), 6.59-7.74(\mathrm{~m}, 22 \mathrm{H}, \mathrm{ArH}), 7.77$ (s, 2H, pyridine-5-H), 7.88-8.09 (m, 6H, ArH). Anal. Calcd. For $\mathrm{C}_{66} \mathrm{H}_{48} \mathrm{~N}_{4} \mathrm{O}_{8} \mathrm{~S}_{4}: \mathrm{C}, 68.73 ; \mathrm{H}, 4.19 ; \mathrm{N}, 4.86 ; \mathrm{S}, 11.12$. Found: $\mathrm{C}, 68.52 ; \mathrm{H}, 4.13 ; \mathrm{N}, 4.69 ; \mathrm{S}, 10.98$.

Synthesis of sodium 3,4-bis(3-(3-amino-4,6-disubstituted thieno[2,3-b]pyridin-2-yl)benzofuran-2-yl)thieno[2,3-b]thiophene-2,5-dicarboxylate 21(a-b). Method A. A mixture of diethyl 3,4-bis((2-(2bromoacetyl)phenoxy)methyl)-thieno[2,3-b]thiophene-2,5-dicarboxylate (18) (5 mmol) in ethanol (25 $\mathrm{mL})$ containing sodium ethoxide (10 mmol) and the appropriate 2-mercapto-4,6-disubstituted nicotinonitriles 3a or $3 \mathbf{b}(10 \mathrm{mmol})$ was added. The reaction mixture was heated at reflux for $5 \mathrm{~h}$. The solid products obtained upon cooling were filtered off and recrystallized from DMF to afford the title compounds $\mathbf{2 1 ( a - b ) . ~}$

Method B. A solution of diethyl 3,4-bis((2-(2-(3-cyano-4,6-disubstitutedpyridin-2-ylthio)acetyl)phenoxy) methyl)thieno[2,3-b]thiophene-2,5-dicarboxylate $19 \mathrm{a}$ or $19 \mathrm{~b}(2 \mathrm{mmol})$ in ethanol $(25 \mathrm{~mL})$ containing sodium ethoxide $(4 \mathrm{mmol}$ ) was heated at reflux for $2 \mathrm{~h}$. The reaction mixture was then cooled, and the solvent was evaporated in vacuo. The solid residue was collected and recrystallized from DMF to afford 21(a-b).

Sodium 3,4-bis(3-(3-amino-4,6-dimethylthieno[2,3-b]pyridin-2-yl)benzofuran-2-yl)thieno[2,3-b]thiophene2,5-dicarboxylate (21a). Yellow powder, $\mathrm{mp}>300{ }^{\circ} \mathrm{C}$; yield (A) 69\%; (B) 67\%, IR (KBr disc) v 3432, 3330, 2960, $1598,1551,1445 \mathrm{~cm}^{-1} .{ }^{1} \mathrm{H}$ NMR $(400 \mathrm{MHz}, \mathrm{DMSO}) \delta 2.48\left(\mathrm{~s}, 6 \mathrm{H}, \mathrm{CH}_{3}\right), 2.74\left(\mathrm{~s}, 6 \mathrm{H}, \mathrm{CH}_{3}\right), 6.77-6.81(\mathrm{~m}, 2 \mathrm{H}, \mathrm{ArH})$, 7.01-7.05 (m, 4H, pyridine-5-H \& ArH), 7.15-7.39 (m, 4H, ArH), 7.79 (br. s, 4H, NH $\mathrm{N}_{2}$. MS (m/z) $856\left(\mathrm{M}^{+}\right)$. Anal. Calcd. For $\mathrm{C}_{42} \mathrm{H}_{26} \mathrm{~N}_{4} \mathrm{Na}_{2} \mathrm{O}_{6} \mathrm{~S}_{4}: \mathrm{C}, 58.87 ; \mathrm{H}, 3.06 ; \mathrm{N}, 6.54 ; \mathrm{S}, 14.97$. Found: $\mathrm{C}, 58.73 ; \mathrm{H}, 2.89 ; \mathrm{N}, 6.43 ; \mathrm{S}, 14.77$.

Sodium 3,4-bis(3-(3-amino-4,6-diphenylthieno[2,3-b]pyridin-2-yl)benzofuran-2-yl)thieno[2,3-b]thiophene2,5-dicarboxylate (21b). Yellow powder, $\mathrm{mp}>300^{\circ} \mathrm{C}$; yield (A) 70\%; (B) 68\%, IR ( $\mathrm{KBr}$ disc) v 3466, 3277, 2918, 1596, 1535, $1442 \mathrm{~cm}^{-1} .{ }^{1} \mathrm{H}$ NMR (300 MHz, DMSO) $\delta 6.76-7.65\left(\mathrm{~m}, 28 \mathrm{H}, \mathrm{NH}_{2} \& \mathrm{ArH}\right), 7.73$ (s, 2H, pyridine-5-H), 8.18-8.20 (m, 4H, ArH). Anal. Calcd. For $\mathrm{C}_{62} \mathrm{H}_{34} \mathrm{~N}_{4} \mathrm{Na}_{2} \mathrm{O}_{6} \mathrm{~S}_{4}: \mathrm{C}, 67.38 ; \mathrm{H}, 3.10 ; \mathrm{N}, 5.07 ; \mathrm{S}, 11.61$. Found: $\mathrm{C}, 67.19$; $\mathrm{H}, 2.95 ; \mathrm{N}, 4.99 ; \mathrm{S}, 11.50$.

Synthesis of 1,1'-(2,2'-(pyridine-2,6-diylbis(methylene))bis(oxy)bis(2,1-phenylene))diethanone (25). A solution of potassium 2-acetylphenolate $(20 \mathrm{mmol})$ and 2,6-bis(bromomethyl)pyridine (7) (10 mmol) in DMF $(20 \mathrm{~mL})$ was heated under reflux for 15 min., during which time, $\mathrm{KBr}$ precipitated. The solvent was then removed in vacuo, and the remaining material was washed with water $(50 \mathrm{~mL})$ and purified by crystallization from Ethanol to give 25.

1,1'-(2,2'-(Pyridine-2,6-diylbis(methylene))bis(oxy)bis(2,1-phenylene))diethanone (25). Pale yellow crystals,

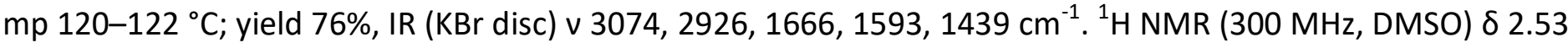
$\left(\mathrm{s}, 6 \mathrm{H}, \mathrm{CH}_{3}\right), 5.29\left(\mathrm{~s}, 4 \mathrm{H}, \mathrm{OCH}_{2}\right), 7.00-7.05(\mathrm{~m}, 2 \mathrm{H}, \mathrm{ArH}), 7.18(\mathrm{~d}, 2 \mathrm{H}, J 8.4 \mathrm{~Hz}), 7.44-7.59(\mathrm{~m}, 6 \mathrm{H}, \mathrm{ArH}), 7.90(\mathrm{t}$, $1 \mathrm{H}, \mathrm{J} 8.1 \mathrm{~Hz})$. MS $(\mathrm{m} / \mathrm{z}) 375\left(\mathrm{M}^{+}\right)$. Anal. Calcd. For $\mathrm{C}_{23} \mathrm{H}_{21} \mathrm{NO}_{4}$ : C, 73.58; H, 5.64; N, 3.73. Found: $\mathrm{C}, 73.40 ; \mathrm{H}$, $5.51 ; \mathrm{N}, 3.65$.

Synthesis of 1,1'-(4,4'-(quinoxaline-2,3-diylbis(methylene))bis(oxy)bis(4,1-phenylene))diethanone (28).

A solution of potassium 4-acetylphenolate $(20 \mathrm{mmol})$ and 2,3-bis(bromomethyl)quinoxaline (27) (10 mmol) in DMF $(20 \mathrm{~mL})$ was heated under reflux for $15 \mathrm{~min}$., during which time, $\mathrm{KBr}$ precipitated. The solvent was then removed in vacuo, and the remaining material was washed with water $(50 \mathrm{~mL})$ and purified by crystallization from ethanol to give 28.

1,1'-(4,4'-(Quinoxaline-2,3-diylbis(methylene))bis(oxy)bis(4,1-phenylene))diethanone (28). Pale yellow crystals, mp $156-158{ }^{\circ} \mathrm{C}$; yield $71 \%$, IR ( $\mathrm{KBr}$ disc) v 3061, 2934, 1670, 1598, $1451 \mathrm{~cm}^{-1} .{ }^{1} \mathrm{H} \mathrm{NMR}(300 \mathrm{MHz}$, DMSO) $\delta 2.49\left(\mathrm{~s}, 6 \mathrm{H}, \mathrm{CH}_{3}\right), 5.67\left(\mathrm{~s}, 4 \mathrm{H}, \mathrm{OCH}_{2}\right), 7.14(\mathrm{~d}, 4 \mathrm{H}, J 8.4 \mathrm{~Hz}), 7.89$ (d, 4H, J $\left.8.4 \mathrm{~Hz}\right), 7.90-7.92(\mathrm{~m}, 2 \mathrm{H}$, quinoxaline-H), 8.10-8.13 (m, $2 \mathrm{H}$, quinoxaline-H). MS $(\mathrm{m} / \mathrm{z}) 426\left(\mathrm{M}^{+}\right)$. Anal. Calcd. For $\mathrm{C}_{26} \mathrm{H}_{22} \mathrm{~N}_{2} \mathrm{O}_{4}: \mathrm{C}, 73.23 ; \mathrm{H}$, 5.20; N, 6.57. Found: C, 73.05; $H, 5.14 ; N, 6.42$. 
Synthesis of (3-amino-4,6-dimethylthieno[2,3-b]pyridin-2-yl)(4-hydroxyphenyl)methanone (31). A mixture of 2-bromo-1-(4-hydroxyphenyl)ethanone (30) $(10 \mathrm{mmol})$ in ethanol $(25 \mathrm{~mL})$ containing sodium ethoxide (10 $\mathrm{mmol}$ ) and 2-mercapto-4,6-dimethylnicotinonitrile $3 \mathrm{a}(10 \mathrm{mmol})$ was added. The reaction mixture was heated at reflux for $5 \mathrm{~h}$. The solid products obtained upon cooling were filtered off and recrystallized from DMF to afford 31.

(3-Amino-4,6-dimethylthieno[2,3-b]pyridin-2-yl)(4-hydroxyphenyl)methanone (31). Yellow powder, $\mathrm{mp} 211-$ $213{ }^{\circ} \mathrm{C}$; yield 82\%, IR ( $\mathrm{KBr}$ disc) v 3482, 3276, 3085, 2967, 1595, $1443 \mathrm{~cm}^{-1} .{ }^{1} \mathrm{H}$ NMR (300 MHz, DMSO) $\delta 2.49(\mathrm{~s}$, $\left.3 \mathrm{H}, \mathrm{CH}_{3}\right), 2.76\left(\mathrm{~s}, 3 \mathrm{H}, \mathrm{CH}_{3}\right), 7.07(\mathrm{~d}, 2 \mathrm{H}, J 8.4 \mathrm{~Hz}), 7.10(\mathrm{~s}, 1 \mathrm{H}$, pyridine-5-H), 7.68 (d, 2H, J $8.4 \mathrm{~Hz}), 7.90$ (br. s, $2 \mathrm{H}$, $\left.\mathrm{NH}_{2}\right), 11.01(\mathrm{~s}, 1 \mathrm{H}, \mathrm{OH})$. MS (m/z) $298\left(\mathrm{M}^{+}\right)$. Anal. Calcd. For $\mathrm{C}_{16} \mathrm{H}_{14} \mathrm{~N}_{2} \mathrm{O}_{2} \mathrm{~S}: \mathrm{C}, 64.41 ; \mathrm{H}, 4.73 ; \mathrm{N}, 9.39 ; \mathrm{S}, 10.75$. Found: $\mathrm{C}, 64.33 ; \mathrm{H}, 4.63 ; \mathrm{N}, 9.23 ; \mathrm{S}, 10.68$.

\section{Supplementary Material}

Supplementary material related to this article, including Nuclear Magnetic Resonance $\left({ }^{1} \mathrm{H}\right.$ and ${ }^{13} \mathrm{C}$ NMR) figures for compounds 4a-d, 5a-d, 8, 9, 10a and b, 11a and b, 14a and b, 15a and b, 16, 19a and b, 21a and b, 25, 28, and $\mathbf{3 1}$ are available in the online version of the text.

\section{References}

1. Lacerda, R. B.; de Lima, C. K. F.; da Silva, L. L.; Romeiro, N. C.; Miranda, A. L. P.; Barreiro, E. J.; Fraga, C. A. M.; Bioorg. Med. Chem. 2009, 17, 74-84.

\section{https://doi.org/10.1016/j.bmc.2008.11.018 http://www.ncbi.nlm.nih.gov/pubmed/19059783.}

2. Duffy, C. D.; Maderna, P.; McCarthy, C.; Loscher, C. E.; Godson, C.; Guiry, P. J.; Chem Med Chem 2010, 5, 517-522.

https://doi.org/10.1002/cmdc.200900533 http://www.ncbi.nlm.nih.gov/pubmed/20127785.

3. Buckley, G. M.; Cooper, N.; Davenport, R. J.; Dyke, H. J.; Galleway, F. P.; Gowers, L.; Haughan, A. F.; Kendall, H. J.; Lowe, C.; Montana, J. G.; Oxford, J.; Peake, J. C.; Picken, C. L.; Richard, M. D.; Sabin, V.; Sharpe, A.; Warneck, J. B. .; Bioorg. Med. Chem. Lett. 2002, 12, 509-512.

\section{https://doi.org/10.1016/S0960-894X(01)00786-7}

4. Vacher, B.; Bonnaud, B.; Funes, P.; Jubault, N.; Koek, W.; Assié, M. B.; Cosi, C.; Kleven, M.; J. Med. Chem. 1999, 42, 1648-1660.

\section{https://doi.org/10.1021/jm9806906}

5. O’Hagan, D.; Nat. Prod. Rep. 2000, 17, 435-446.

https://doi.org/10.1039/a707613d

6. Horiuch, M.; Murakami, C.; Fukamiya, N.; Yu, D.; Chen, T. H.; Bastow, K. F.; Zhang, D. C.; Takaishi, Y.; Imakura, Y.; Lee, K. H.; J. Nat. Prod. 2006, 69, 1271-1274.

https://doi.org/10.1021/np060124a http://www.ncbi.nlm.nih.gov/pubmed/16989518

7. Song, Z. J.; Zhao, M.; Desmond, R.; Devine, P.; Tschaen, D. M.; Tillyer, R.; Frey, L.; Heid, R.; Xu, F.; Foster, B.; Li, J.; Reamer, R.; Volante, R.; Grabowski, E. J. J.; Dolling, U. H.; Reider, P. J.; Okada, S.; Kato, Y.; Mano, E.; J. Org. Chem. 1999, 64, 9658-9667.

https://doi.org/10.1021/jo991292t

8. Abe, Y.; Kayakiri, H.; Satoh, S.; Inoue, T.; Sawada, Y.; Inamura, N.; Asano, M.; Aramori, I.; Hatori, C.; Sawai, 
H.; Oku, T.; Tanaka, H.; J. Med. Chem. 1998, 41, 4062-4079.

https://doi.org/10.1021/jm980300f

9. Lu, C. M.; Chen, Y. L.; Chen, H. L.; Chen, C. A.; Lu, P. J.; Yang, C. N.; Tzeng, C. C.; Bioorganic Med. Chem. 2010, 18, 1948-1957.

https://doi.org/10.1016/j.bmc.2010.01.033

10. Ren, Q.; Mo, W.; Gao, L.; He, H.; Gu, Y.; J. Heterocycl. Chem. 2010, 47, 171-178. https://doi.org/10.1002/jhet.296

11. Zhang, W.; Chen, Y.; Chen, W.; Liu, Z.; Li, Z.; J. Agric. Food Chem. 2010, 58, 6296-6299. https://doi.org/10.1021/jf100645y

12. Zhao, X. H.; Han, C. M.; Li, Y.; Bai, M. G.; Yang, J. C.; Xu, H.; Yuan, S. D.; Xie, L. H.; Xu, Z. J.; Dye. Pigment. 2020, 175, 108127.

https://doi.org/10.1016/j.dyepig.2019.108127

13. Bull, J. A.; Mousseau, J. J.; Pelletier, G.; Charette, A. B.; Chem. Rev. 2012, 112, 2642-2713. https://doi.org/10.1021/cr200251d http://www.ncbi.nlm.nih.gov/pubmed/22352938

14. Allais, C.; Grassot, J. M.; Rodriguez, J.; Constantieux, T.; Chem. Rev. 2014, 114, 10829-10868. https://doi.org/10.1021/cr500099b

15. Lu, N.; Li, L.; Zheng, X.; Zhang, S.; Li, Y.; Yuan, J.; Wei, Q.; Xu, Y.; Meng, F.; Molecules 2018, $23,1041$. https://doi.org/10.3390/molecules23051041

16. van Rensburg, M.; Leung, E.; Haverkate, N. A.; Eurtivong, C.; Pilkington, L. I.; Reynisson, J.; Barker, D.; Bioorganic Med. Chem. Lett. 2017, 27, 135-138. https://doi.org/10.1016/j.bmcl.2016.12.009 http://www.ncbi.nlm.nih.gov/pubmed/27979592

17. Chaubey, A.; Pandeya, S. N.; Asian J. Pharm. Clin. Res. 2011, 4, 5-8.

18. Lang, X.; Li, L.; Chen, Y.; Sun, Q.; Wu, Q.; Liu, F.; Tan, C.; Liu, H.; Gao, C.; Jiang, Y.; Bioorganic Med. Chem. 2013, 21, 4170-4177.

https://doi.org/10.1016/j.bmc.2013.05.008 http://www.ncbi.nlm.nih.gov/pubmed/23735826

19. Al-Trawneh, S. A.; El-Abadelah, M. M.; Zahra, J. A.; Al-Taweel, S. A.; Zani, F.; Incerti, M.; Cavazzoni, A.; Vicini, P.; Bioorg. Med. Chem. 2011, 19, 2541-2548.

https://doi.org/10.1016/j.bmc.2011.03.018 http://www.ncbi.nlm.nih.gov/pubmed/21458275

20. Kamata, M.; Yamashita, T.; Kina, A.; Funata, M.; Mizukami, A.; Sasaki, M.; Tani, A.; Funami, M.; Amano, N.; Fukatsu, K.; Bioorg. Med. Chem. Lett. 2012, 22, 3643-3647.

https://doi.org/10.1016/j.bmcl.2012.04.047

21. Ueda, M.; Matsumura, S.; Masui, M.; Matsuura, E.; Kawakami, M.; Fujitomo, H.; Umeda, T.; Kagawa, H.; Hirohata, S.; Shima, K.; Arzneimittelforschung. 1993, 43, 1282-1290.

http://www.ncbi.nlm.nih.gov/pubmed/8141815

22. Saito, K.; Nakao, A.; Shinozuka, T.; Shimada, K.; Matsui, S.; Oizumi, K.; Yano, K.; Ohata, K.; Nakai, D.; Nagai, Y.; Naito, S.; Bioorg. Med. Chem. 2013, 21, 1628-1642.

https://doi.org/10.1016/j.bmc.2013.01.071

23. Lockman, J. W.; Reeder, M. D.; Suzuki, K.; Ostanin, K.; Hoff, R.; Bhoite, L.; Austin, H.; Baichwal, V.; Adam Willardsen, J.; Bioorganic Med. Chem. Lett. 2010, 20, 2283-2286.

https://doi.org/10.1016/j.bmcl.2010.02.005

24. Feng, L.; Reynisdóttir, I.; Reynisson, J.; Eur. J. Med. Chem. 2012, 54, 463-469. https://doi.org/10.1016/j.ejmech.2012.05.029 http://www.ncbi.nlm.nih.gov/pubmed/22698703

25. Dai, X.-Y.; Zeng, X.-X.; Peng, F.; Han, Y.-Y.; Lin, H.-J.; Xu, Y.-Z.; Zhou, T.; Xie, G.; Deng, Y.; Mao, Y.-Q.; Yu, L.T.; Yang, L.; Zhao, Y.-L.; Cell. Physiol. Biochem. 2012, 29, 281-290. 
26. Jain, M.; Sakhuja, R.; Khanna, P.; Bhagat, S.; Jain, S.; Arkivoc 2008, xv, 54-64.

27. Wang, Z.; Zhao, C.; Zhao, D.; Li, C.; Zhang, J.; Wang, H.; Tetrahedron 2010, 66, 2168-2174. https://doi.org/10.1016/j.tet.2010.01.056

28. Li, J. T.; Sun, M. X.; He, G. Y.; Xu, X. Y.; Ultrason. Sonochem. 2011, 18, 412-414. https://doi.org/10.1016/j.ultsonch.2010.07.016

29. Shaveta; Mishra, S.; Singh, P.; Eur. J. Med. Chem. 2016, 124, 500-536. https://doi.org/10.1016/i.ejmech.2016.08.039 http://www.ncbi.nlm.nih.gov/pubmed/27598238

30. Pertino, M. W.; Petrera, E.; Edith Alché, L.; Schmeda-Hirschmann, G.; Molecules 2018, 23, 1343. https://doi.org/10.3390/molecules23061343

31. Tiwari, S. V.; Seijas, J. A.; Vazquez-Tato, M. P.; Sarkate, A. P.; Karnik, K. S.; Nikalje, A. P. G.; Molecules 2018, $23,440$.

https://doi.org/10.3390/molecules23020440

32. Abd El-Fatah, N. A.; Darweesh, A. F.; Mohamed, A. A.; Abdelhamid, I. A.; Elwahy, A. H. M.; Tetrahedron 2017, 73, 1436-1450.

https://doi.org/10.1016/j.tet.2017.01.047

33. Muathen, H. A.; Aloweiny N. A. M.; Elwahy, A. H. M.; J. Heterocycl. Chem. 2009, 46(4), 656-663. https://doi.org/10.1002/jhet.129

34. Salem, M. E.; Darweesh, A. F.; Elwahy, A. H. M.; J. Sulfur Chem. 2018, 39, 525-543. https://doi.org/10.1080/17415993.2018.1471143

35. M. Abdella, A.; H. M. Elwahy, A.; A. Abdelhamid, I.; Curr. Org. Synth. 2016, 13, 601-610. https://doi.org/10.2174/1570179413999151211115100

36. Salama, S. K.; Mohamed, M. F.; Darweesh, A. F.; Elwahy, A. H. M.; Abdelhamid, I. A.; Bioorg. Chem. 2017, 71, 19-29.

https://doi.org/10.1016/j.bioorg.2017.01.009

37. Salem, M. E.; Ahmed, A. A.; Darweesh, A. F.; Kühn, O.; Elwahy, A.H.M., J. Mol. Struc. 2019, 1176, $19-30$. https://doi.org/10.1016/j.molstruc.2018.08.031

38. Salem, M. E.; Darweesh, A. F.; Mekky, A. E. M.; Farag, A. M.; Elwahy, A. H. M.; J. Heterocycl. Chem. 2017, 54, 226-234.

https://doi.org/10.1002/jhet.2571

39. Abd El-Fatah, N. A.; Darweesh, A. F.; Mohamed, A. A.; Abdelhamid, I. A.; Elwahy, A. H. M.; Monatshefte fur Chemie 2017, 148, 2107-2122.

https://doi.org/10.1007/s00706-017-2040-7

40. Ginidi, A. R. S.; Shaaban, M. R.; Farag, A. M.; Elwahy, A. H. M.; J. Heterocycl. Chem. 2015, 52, 1421-1428. https://doi.org/10.1002/jhet.2223

41. Sayed, O. M.; Mekky, A. E. M.; Farag, A. M.; Elwahy, A. H. M.; J. Sulfur Chem. 2015, 36, 124-134. https://doi.org/10.1080/17415993.2014.975131

42. Elwahy, A. H. M.; Tetrahedron 2000, 56, 897-907. https://doi.org/10.1016/S0040-4020(99)01072-8

43. Elwahy, A. H. M.; Abbas, A. A.; Tetrahedron 2000, 56, 885-895. https://doi.org/10.1016/S0040-4020(99)01068-6

44. Hu, K.; Jeong, J. H.; Arch. Pharm. Res. 2006, 29, 476-478. https://doi.org/10.1007/BF02969419

45. Chen, Y. Y.; Zhang, N. N.; Ye, L. M.; Chen, J. H.; Sun, X.; Zhang, X. J.; Yan, M.; RSC Adv. 2015, 5, 4804648049. 
https://doi.org/10.1039/c5ra07188g

46. Dyachenko, V. D.; Dyachenko, A. D.; Chernega, A. N.; Russ. J. Org. Chem. 2004, 40, 397-406. https://doi.org/10.1023/B:RUJO.0000034978.81993.bd

This paper is an open access article distributed under the terms of the Creative Commons Attribution (CC BY) license (http://creativecommons.org/licenses/by/4.0/) 